\title{
Weak-type operators and the strong fundamental lemma of real interpolation theory
}

\author{
by \\ N. Krugluak (Luleå), Y. Sagher (Boca Raton, FL) \\ and P. Shvartsman (Haifa)
}

\begin{abstract}
We prove an interpolation theorem for weak-type operators. This is closely related to interpolation between weak-type classes. Weak-type classes at the ends of interpolation scales play a similar role to that played by BMO with respect to the $L^{p}$ interpolation scale. We also clarify the roles of some of the parameters appearing in the definition of the weak-type classes. The interpolation theorem follows from a $K$-functional inequality for the operators, involving the Calderón operator. The inequality was inspired by a $K-J$ inequality approach developed by Jawerth and Milman. We show that the use of the Calderón operator is necessary. We use a new version of the strong fundamental lemma of interpolation theory that does not require the interpolation couple to be mutually closed.
\end{abstract}

1. Introduction. Weak-type classes were defined in [9], [10], and [11]. Interpolation theorems between these classes give stronger versions of classical interpolation theorems, and calculations of the $K$-functionals between these classes give a systematic way of proving rearrangement-function inequalities for classical operators. These classes, when constructed at the natural ends of interpolation scales, turn out to be interesting in their own right, and imply useful generalizations of interpolation theorems. The definition of the weak-type classes, Definition 1.2 below, is quite general, and among other things yields an interpolation theorem, Theorem 1.3, that is valid only for a subinterval of the interpolation scale.

In this paper we generalize the interpolation theorem. We define weaktype operators between interpolation pairs of Banach groups, and prove an interpolation theorem for these operators. Moreover we prove an inequality between the $K$-functionals of $T a$ and of $a$, which implies the interpolation theorem and is of independent interest. We also show that the interpolation

2000 Mathematics Subject Classification: Primary 46B70.

Key words and phrases: interpolation theory, weak-type operators, strong fundamental lemma. 
theorem between weak-type classes is a special case of the new interpolation theorem, and clarify the subinterval phenomenon in Theorem 1.3.

Let us recall some of the notions. Let $\bar{A}=\left(A_{0}, A_{1}\right)$ be an interpolation couple. Here $A_{j}$ are quasi-Banach groups, that is to say, there exist functions $\|\cdot\|_{A_{j}}: A_{j} \rightarrow \mathbb{R}_{+}$so that $\|a\|_{A_{j}}=0 \Leftrightarrow a=0,\|a\|_{A_{j}}=\|-a\|_{A_{j}}$ and $\|\cdot\|_{A_{j}}$ satisfies the quasi-triangle inequality:

$$
\|a+b\|_{A_{j}} \leq c_{j}\left(\|a\|_{A_{j}}+\|b\|_{A_{j}}\right) .
$$

Let

$$
K(t, a ; \bar{A})=\inf \left\{\left\|a_{0}\right\|_{A_{0}}+t\left\|a_{1}\right\|_{A_{1}}: a_{0}+a_{1}=a, a_{j} \in A_{j}\right\} .
$$

Definition 1.1. Let $\bar{A}=\left(A_{0}, A_{1}\right)$ and $\bar{B}=\left(B_{0}, B_{1}\right)$ be two interpolation couples of quasi-Banach groups. An operator

$$
T: A_{0} \cap A_{1} \rightarrow B_{0}+B_{1}
$$

is said to be $K$-subadditive if there is a constant $M$ so that for all $a_{0}, a_{1} \in$ $A_{0} \cap A_{1}$ and all $t>0$,

$$
K\left(t, T\left(a_{0}+a_{1}\right) ; \bar{B}\right) \leq M\left(K\left(t, T a_{0} ; \bar{B}\right)+K\left(t, T a_{1} ; \bar{B}\right)\right) .
$$

It is easy to see that if $B_{0}$ and $B_{1}$ are Banach lattices and for almost every $\omega$,

$$
\left|T\left(a_{0}+a_{1}\right)(\omega)\right| \leq M\left(\left|T a_{0}(\omega)\right|+\left|T a_{1}(\omega)\right|\right),
$$

then (1.1) holds.

We denote by $\widehat{\phi}$ the least concave majorant of a function $\phi: \mathbb{R}_{+} \rightarrow \mathbb{R}_{+}$.

We let $c$ denote a generic constant which depends on parameters that are fixed in the context. Also: if $f$ and $g$ are two non-negative functions and there exists a $c>0$ so that $c^{-1} f \leq g \leq c f$, we write $f \backsim g$.

We will also use the following notation: if $g: \mathbb{R}_{+} \rightarrow \mathbb{R}_{+}$,

$$
g_{T}(t)=\operatorname{tg}(1 / t) \text {. }
$$

Recall that

$$
K_{T}\left(\cdot, a ; A_{0}, A_{1}\right)=K\left(\cdot, a ; A_{1}, A_{0}\right) .
$$

If $\bar{A}=\left(A_{0}, A_{1}\right)$, we will denote by $\bar{A}^{T}$ the interpolation couple $\left(A_{1}, A_{0}\right)$.

Definition 1.2. Let $\bar{A}=\left(A_{0}, A_{1}\right)$ be an interpolation couple. Let

$$
g: \mathbb{R}_{+} \times\left(A_{0}+A_{1}\right) \rightarrow \mathbb{R}_{+}
$$

be such that

$$
\widehat{g} \backsim K(\cdot, \cdot ; \bar{A}) .
$$

For $0 \leq \varepsilon<\infty, 0<r<\infty$, and $1<\gamma<\infty$, we define

$$
\|a\|_{W_{K}[\bar{A} ; \varepsilon, \gamma, r, g]}=\sup _{t>0}\left(\left[g^{r}(\gamma t, a)-\varepsilon^{r} g^{r}(t, a)\right]_{+}^{1 / r}\right)
$$


and

$$
W_{K}[\bar{A} ; \varepsilon, \gamma, r, g]=\left\{a \in A_{0}+A_{1}:\|a\|_{W_{K}[\bar{A} ; \varepsilon, \gamma, r, g]}<\infty\right\} .
$$

Recall (see Theorem 3.6 in [9]) that $(\widehat{g})_{T}=\left(g_{T}\right)^{\frown}$. Moreover, (1.4) is equivalent to

$$
\left(g_{T}\right)^{\frown} \backsim K\left(\cdot, \cdot ; A_{1}, A_{0}\right)
$$

so that if $W_{K}[\bar{A} ; \varepsilon, \gamma, r, g]$ is defined, then so is $W_{K}\left[\bar{A}^{T} ; \varepsilon, \gamma, r, g_{T}\right]$.

The large number of parameters in the definition of $W_{K}$ is forced on us by the formulas of interpolation theory. In most cases the $K$-functional can be calculated only up to equivalence. For example Holmstedt's Theorem, which plays a central role in the theory, gives an expression which is equivalent to a $K$-functional. Since the definition of $W_{K}$ involves differences, we cannot replace functions by other, equivalent ones, in the definition, without changing the conclusions of the theorems. The parameters $g$ and $r$ solve this problem.

The following interpolation theorem was proved in [9] for the case of Banach groups.

Theorem 1.3. Let $\bar{A}=\left(A_{0}, A_{1}\right)$ and $\bar{B}=\left(B_{0}, B_{1}\right)$ be two interpolation couples. Let

$$
T: A_{0} \cap A_{1} \rightarrow B_{0}+B_{1}
$$

be a $K$-subadditive operator. Assume that $\hat{g} \backsim K(\cdot, \cdot ; \bar{B})$ and that for all $a \in A_{0} \cap A_{1}$,

$$
\|T a\|_{W_{K}\left[\bar{B} ; \varepsilon_{0}, \gamma, r, g\right]} \leq M_{0}\|a\|_{A_{0}}
$$

and

$$
\|T a\|_{W_{K}\left[\bar{B}^{T} ; \varepsilon_{1}, \gamma, r, g_{T}\right]} \leq M_{1}\|a\|_{A_{1}}
$$

where $0<r<\infty, \gamma>1,0 \leq \varepsilon_{0}, \varepsilon_{1}<\gamma$ and $\varepsilon_{0} \varepsilon_{1}<\gamma$. Then for all $\theta$ so that

$$
\log _{\gamma}^{+} \varepsilon_{0}<\theta<1-\log _{\gamma}^{+} \varepsilon_{1}
$$

and $0<q \leq \infty$, we have

$$
\|T a\|_{\bar{B}_{\theta, q}} \leq C M_{0}^{1-\theta} M_{1}^{\theta}\|a\|_{\bar{A}_{\theta, q}}
$$

where $C$ is a constant that depends on the parameters defining $W_{K}$ and on $\theta, q$.

We will see below that Theorem 1.3 follows from a more general interpolation theorem. The new theorem also clarifies the restriction on $\theta$ given in (1.7).

Set

$$
\theta_{0}=\log _{\gamma}^{+} \varepsilon_{0}, \quad \theta_{1}=1-\log _{\gamma}^{+} \varepsilon_{1}, \quad G(t, b)=t^{-\theta_{0} /\left(\theta_{1}-\theta_{0}\right)} g\left(t^{1 /\left(\theta_{1}-\theta_{0}\right)}, b\right) .
$$


Write $F=\widehat{G}$. We will see that

$$
F(t, b) \backsim K\left(t, b ; \bar{B}_{\theta_{0}, \infty}, \bar{B}_{\theta_{1}, \infty}\right)
$$

where $\bar{B}_{\theta_{0}, \infty}$ is replaced by $B_{0}$ when $\theta_{0}=0$, and $\bar{B}_{\theta_{1}, \infty}$ is replaced by $B_{1}$ when $\theta_{1}=1$. Observe that, by (1.3), (1.9) is equivalent to

$$
F_{T}(t, b) \backsim K\left(t, b ; \bar{B}_{\theta_{1}, \infty}, \bar{B}_{\theta_{0}, \infty}\right) .
$$

Define

$$
\lambda=\gamma^{\theta_{1}-\theta_{0}}, \quad \sigma_{0}=\frac{\theta_{0}}{\theta_{1}-\theta_{0}}, \quad \sigma_{1}=\frac{1-\theta_{1}}{\theta_{1}-\theta_{0}} .
$$

A simple calculation shows that condition (1.5) is equivalent to

$$
\sup _{t>0}\left(t^{\sigma_{0}}\left[G^{r}(\lambda t, T a)-G^{r}(t, T a)\right]_{+}^{1 / r}\right) \leq c M_{0}\|a\|_{A_{0}}
$$

and condition (1.6) is equivalent to

$$
\sup _{t>0}\left(t^{\sigma_{1}}\left[G_{T}^{r}(\lambda t, T a)-G_{T}^{r}(t, T a)\right]_{+}^{1 / r}\right) \leq c M_{1}\|a\|_{A_{1}} .
$$

We shall prove that (1.10) implies

$$
\sup _{t>0}\left(t^{\sigma_{0}}\left(t\left(F^{r}\right)^{\prime}(t, T a)\right)^{1 / r}\right) \leq c M_{0}\|a\|_{A_{0}}
$$

and similarly (1.11) yields

$$
\sup _{t>0}\left(t^{\sigma_{1}}\left(t\left(F_{T}^{r}\right)^{\prime}(t, T a)\right)^{1 / r}\right) \leq c M_{1}\|a\|_{A_{1}} .
$$

We shall then show that (1.12) and (1.13) imply that for some $p$,

$$
K\left(t, T a ; \bar{B}_{\theta_{0}, \infty}, \bar{B}_{\theta_{1}, \infty}\right) \leq c S_{p}\left(K\left(\cdot, a ; \bar{A}_{\theta_{0}, \infty}, \bar{A}_{\theta_{1}, \infty}\right)\right)(t)
$$

where $S_{p}$ is a Calderón operator defined below (see (2.4)). Finally, we show that (1.14) implies the interpolation result (1.8) (see Theorem 2.6).

Recall that $F$, and of course also $F_{T}$, is a positive concave function on $\mathbb{R}_{+}$and so is absolutely continuous. It may fail to have a derivative on a countable set of points and since our inequalities are pointwise let us agree that $F^{\prime}$ and $\left(F_{T}\right)^{\prime}$ stand for the right derivatives at these points.

Our approach is based on an application of a version of the strong fundamental lemma of interpolation theory to prove that the weak-type conditions imply (1.14). This approach was initiated by Jawerth and Milman in $[4$, pp. $49-50]$.

We will assume the standard results of interpolation theory as stated in $[1]$.

2. Weak-type operators. Conditions (1.12) and (1.13) motivate the following definition. 
Definition 2.1. Let $\bar{A}=\left(A_{0}, A_{1}\right)$ and $\bar{B}=\left(B_{0}, B_{1}\right)$ be two interpolation couples of quasi-Banach groups. Let $\sigma_{0}, \sigma_{1} \geq 0$ and $r>0$. A $K$-subadditive operator $T$ is said to be an $\bar{A} \rightarrow \bar{B}$ weak-type $\left(\sigma_{0}, \sigma_{1} ; r\right)$ operator if there exists a function

$$
F: \mathbb{R}_{+} \times\left(B_{0}+B_{1}\right) \rightarrow \mathbb{R}_{+}
$$

concave in the first argument so that $K(\cdot, \cdot ; \bar{B}) \leq F$ and for some $0<$ $M_{0}, M_{1}<\infty$ and $a \in A_{0} \cap A_{1}$,

$$
\sup _{t>0}\left(t^{\sigma_{0}}\left(t\left(F^{r}\right)^{\prime}(t, T a)\right)^{1 / r}\right) \leq M_{0}\|a\|_{A_{0}}
$$

and

$$
\sup _{t>0}\left(t^{\sigma_{1}}\left(t\left(F_{T}^{r}\right)^{\prime}(t, T a)\right)^{1 / r}\right) \leq M_{1}\|a\|_{A_{1}} .
$$

The numbers $M_{0}$ and $M_{1}$ are called the norms of the operator.

REMARK 2.2. Observe that $T$ is an $\bar{A} \rightarrow \bar{B}$ weak-type $\left(\sigma_{0}, \sigma_{1} ; r\right)$ operator iff it is an $\bar{A}^{T} \rightarrow \bar{B}^{T}$ weak-type $\left(\sigma_{1}, \sigma_{0} ; r\right)$ operator.

Condition (2.2) can also be written

$$
\sup _{t>0}\left(t^{-\sigma_{1}}\left(-t\left(\frac{F^{r}(t, T a)}{t^{r}}\right)^{\prime}\right)^{1 / r}\right) \leq M_{1}\|a\|_{A_{1}} .
$$

Weak-type operators, with $\sigma_{0}=\sigma_{1}=0, F=K$, and $r=1$, were introduced in $[8]$.

In practice we frequently get a function, $G$, so that $K \leq \widehat{G}$, and to see that $T$ is a weak-type $\left(\sigma_{0}, \sigma_{1} ; r\right)$ operator we want to see if $F:=\widehat{G}$ satisfies (2.1) and (2.2). This could be hard since it involves precise calculations of $\widehat{G}$ and its derivative. We will see in Section 3 that it suffices to verify that $G$ satisfies conditions (1.10) and (1.11).

Let us recall the definition of Calderón operators.

Definition 2.3. For $p>0$, we define the Calderón operator acting on non-negative functions on $\mathbb{R}_{+}$by the formula

$$
S_{p} f(t)=\left(\int_{0}^{\infty}\left(\min \left\{1, \frac{t}{s}\right\} f(s)\right)^{p} \frac{d s}{s}\right)^{1 / p} .
$$

If $p=1$ we have the usual Calderón operator. In this case we write $S$ rather than $S_{1}$.

We will need the following 
Lemma 2.4. Assume that $T: A_{0} \cap A_{1} \rightarrow B_{0}+B_{1}$ is a $K$-subadditive operator (see (1.1)). Then, for all $u_{j} \in A_{0} \cap A_{1}$ and all $t>0$,

$$
K\left(t, T\left(\sum_{j=1}^{n} u_{j}\right) ; \bar{B}\right) \leq 2 M\left(\sum_{j=1}^{n} K^{\alpha}\left(t, T u_{j} ; \bar{B}\right)\right)^{1 / \alpha}
$$

where

$$
\alpha=\frac{1}{1+\log _{2} M} .
$$

Proof. We consider each $K(t, T \cdot ; \bar{B})$ as a seminorm on the group $A_{0} \cap A_{1}$ and apply the Aoki-Rolewicz Theorem (see e.g. [6]).

The letter $\alpha$ from now on stands for the quantity in (2.6).

Our main goal is to prove the following theorem.

TheOREM 2.5. If $T: \bar{A} \rightarrow \bar{B}$ is a weak-type $\left(\sigma_{0}, \sigma_{1} ; r\right)$ operator with norms $M_{0}, M_{1}$, then for all $\sigma_{0}, \sigma_{1} \geq 0, a \in A_{0} \cap A_{1}$ and $t>0$,

$$
K(t, T a ; \bar{B}) \leq c M_{0} S_{\min \{\alpha, r\}}\left[K\left(\cdot, a ; \bar{A}_{\theta_{0}, \infty}, \bar{A}_{\theta_{1}, \infty}\right)\right]\left(\frac{M_{1}}{M_{0}} t\right)
$$

where

$$
\begin{aligned}
& \theta_{0}=\frac{\sigma_{0}}{1+\sigma_{0}+\sigma_{1}}, \\
& \theta_{1}=\frac{1+\sigma_{0}}{1+\sigma_{0}+\sigma_{1}} .
\end{aligned}
$$

If $\sigma_{0}=0$ we replace $\bar{A}_{\theta_{0}, \infty}$ by $A_{0}$. If $\sigma_{1}=0$ we replace $\bar{A}_{\theta_{1}, \infty}$ by $A_{1}$.

If $\sigma_{0}>0$ and $\sigma_{1}>0$ we get a somewhat stronger result, with $S_{\alpha}$ instead of $S_{\min \{\alpha, r\}}$ in (2.7).

In Theorem 3.5 we give an example of a weak-type $\left(\sigma_{0}, \sigma_{1} ; r\right)$ operator. Also, in the discussion which follows Theorem 3.5 we show that in some cases (2.7) is sharp, that is, there exists a class of functions for which we can replace the inequality in (2.7) by an equivalence.

Applying Hardy's inequalities (and their extensions for quasi-monotone functions to get the case $0<q<1$ below) to (2.7) we get the following interpolation theorem:

TheOREM 2.6. If $T: \bar{A} \rightarrow \bar{B}$ is a weak-type $\left(\sigma_{0}, \sigma_{1} ; r\right)$ operator with norms $M_{0}, M_{1}$, where $\sigma_{0}, \sigma_{1} \geq 0$, then for $\theta_{0}, \theta_{1}$ given by (2.8) and (2.9), and all $a \in A_{0} \cap A_{1}, 0<\eta<1,0<q \leq \infty$,

$$
\|T a\|_{\bar{B}_{\eta, q}} \leq c M_{0}^{1-\eta} M_{1}^{\eta}\|a\|_{\bar{A}_{(1-\eta) \theta_{0}+\eta \theta_{1}, q}} .
$$

We return to Theorem 2.5. Renorming $A_{0}$ and $A_{1}$, we can assume that $M_{0}=M_{1}=1$. We will also write $F(t)$ for $F(t, T a)$. 
For the proof of the theorem we will need some preliminary lemmas. To state the first lemma we need to define the following function.

Definition 2.7. For $x>0$ and $\sigma_{0}, \sigma_{1} \geq 0$ we define

$$
\Psi\left(x, \sigma_{0}, \sigma_{1}\right)= \begin{cases}x & \text { if } x \leq 1 \text { and } \sigma_{1}>0, \\ x(1+|\log x|) & \text { if } x \leq 1 \text { and } \sigma_{1}=0, \\ 1 & \text { if } 1<x \text { and } \sigma_{0}>0 \\ 1+|\log x| & \text { if } 1<x \text { and } \sigma_{0}=0 .\end{cases}
$$

Observe that

$$
\Psi\left(x, \sigma_{0}, \sigma_{1}\right)=\Psi_{T}\left(x, \sigma_{1}, \sigma_{0}\right)
$$

and that for $q \geq p>0$,

$$
\Psi^{1 / q}\left(x^{q}, \sigma_{0}, \sigma_{1}\right) \leq \Psi^{1 / p}\left(x^{p}, \sigma_{0}, \sigma_{1}\right) .
$$

Lemma 2.8. Set

$$
h\left(s, \sigma_{0}, \sigma_{1}\right)=s^{-\sigma_{0}} \min \left\{1, s^{1+\sigma_{0}+\sigma_{1}}\right\} .
$$

For $\sigma_{0}, \sigma_{1} \geq 0$,

$$
\Psi\left(\cdot, \sigma_{0}, \sigma_{1}\right) \backsim \operatorname{Sh}\left(\cdot, \sigma_{0}, \sigma_{1}\right)(\cdot) .
$$

Proof. For $0<x \leq 1$ and $\sigma_{1}>0$ we have

$$
\begin{aligned}
\operatorname{Sh}\left(\cdot, \sigma_{0}, \sigma_{1}\right)(x) & =\int_{0}^{\infty} \min \left\{1, \frac{x}{s}\right\} s^{-\sigma_{0}} \min \left\{1, s^{1+\sigma_{0}+\sigma_{1}}\right\} \frac{d s}{s} \\
& =\int_{0}^{x} s^{1+\sigma_{1}} \frac{d s}{s}+x \int_{x}^{1} s^{\sigma_{1}} \frac{d s}{s}+x \int_{1}^{\infty} s^{-\left(1+\sigma_{0}\right)} \frac{d s}{s} \\
& \sim x=\Psi\left(x, \sigma_{0}, \sigma_{1}\right) .
\end{aligned}
$$

If $0<x \leq 1$ and $\sigma_{1}=0$ then

$$
\begin{aligned}
S h\left(\cdot, \sigma_{0}, 0\right)(x) & =x+x \log \frac{1}{x}+\frac{1+\sigma_{1}}{1+\sigma_{0}+\sigma_{1}} x \\
& \backsim x(1+|\log x|)=\Psi\left(x, \sigma_{0}, 0\right) .
\end{aligned}
$$

It is easy to verify that $S\left(f_{T}\right)=(S f)_{T}$ and that

$$
h_{T}\left(\cdot, \sigma_{1}, \sigma_{0}\right)(t)=h\left(\cdot, \sigma_{0}, \sigma_{1}\right)(t),
$$

which together with (2.10) implies (2.12) also in the case $x>1$.

Lemma 2.9. Let $\sigma_{0}, \sigma_{1} \geq 0$. If $T$ is an $\bar{A} \rightarrow \bar{B}$ weak-type $\left(\sigma_{0}, \sigma_{1} ; r\right)$ operator with norms $M_{0}=M_{1}=1$ then for any $a \in A_{0} \cap A_{1}$ and any $s, t>0$, we have

$$
K(t, T a ; \bar{B}) \leq c \Psi^{1 / r}\left((t / s)^{r}, \sigma_{0}, \sigma_{1}\right) s^{-\sigma_{0}} J\left(s^{1+\sigma_{0}+\sigma_{1}}, a ; \bar{A}\right) .
$$


Proof. Let us consider the case $t \leq s$ and $\sigma_{1}>0$. Then, by (2.3),

$$
\left(\frac{F(t)}{t}\right)^{r}-\left(\frac{F(s)}{s}\right)^{r}=-\int_{t}^{s}\left(\frac{F^{r}(\tau)}{\tau^{r}}\right)^{\prime} d \tau \leq\|a\|_{A_{1}}^{r} \int_{t}^{s} \tau^{-1+r \sigma_{1}} d \tau
$$

so that

$$
\left(\frac{F(t)}{t}\right)^{r}-\left(\frac{F(s)}{s}\right)^{r} \leq c\|a\|_{A_{1}}^{r} s^{r \sigma_{1}} .
$$

But (2.3) can also be written

$$
-\frac{\left(F^{r}\right)^{\prime}(s)}{s^{r}}+r \frac{F^{r}(s)}{s^{r+1}} \leq\|a\|_{A_{1}}^{r} s^{-1+r \sigma_{1}}
$$

so that

$$
\left(\frac{F(s)}{s}\right)^{r} \leq \frac{1}{r}\|a\|_{A_{1}}^{r} s^{r \sigma_{1}}+\frac{1}{r} \frac{\left(F^{r}\right)^{\prime}(s)}{s^{r-1}}
$$

By $(2.1)$,

$$
\begin{aligned}
\left(\frac{F(s)}{s}\right)^{r} & \leq \frac{1}{r}\|a\|_{A_{1}}^{r} s^{r \sigma_{1}}+\frac{1}{r}\|a\|_{A_{0}}^{r} s^{-1-r \sigma_{0}} s^{1-r} \\
& =\frac{1}{r}\|a\|_{A_{1}}^{r} s^{r \sigma_{1}}+\frac{1}{r}\|a\|_{A_{0}}^{r} s^{-r\left(1+\sigma_{0}\right)} .
\end{aligned}
$$

By $(2.15)$ for $t \leq s$,

$$
\left(\frac{F(t)}{t}\right)^{r} \leq\left(\frac{F(s)}{s}\right)^{r}+c\|a\|_{A_{1}}^{r} s^{r \sigma_{1}} \leq c\left(\|a\|_{A_{0}}^{r} s^{-r\left(1+\sigma_{0}\right)}+\|a\|_{A_{1}}^{r} s^{r \sigma_{1}}\right)
$$

so that

$$
\begin{aligned}
F^{r}(t) & \leq c t^{r} s^{-r\left(1+\sigma_{0}\right)}\left(\|a\|_{A_{0}}^{r}+\|a\|_{A_{1}}^{r} s^{r\left(1+\sigma_{0}+\sigma_{1}\right)}\right) \\
& =c(t / s)^{r} s^{-r \sigma_{0}}\left(\|a\|_{A_{0}}^{r}+\|a\|_{A_{1}}^{r} s^{r\left(1+\sigma_{0}+\sigma_{1}\right)}\right) \\
& \leq c(t / s)^{r} s^{-r \sigma_{0}} J^{r}\left(s^{1+\sigma_{0}+\sigma_{1}}, a ; \bar{A}\right) \\
& =c \Psi\left((t / s)^{r}, \sigma_{0}, \sigma_{1}\right) s^{-r \sigma_{0}} J^{r}\left(s^{1+\sigma_{0}+\sigma_{1}}, a ; \bar{A}\right)
\end{aligned}
$$

and since $K \leq F$ we proved (2.13) for $t \leq s$ and $\sigma_{1}>0$.

Let us consider the case $t \leq s$ when $\sigma_{1}=0$. Estimate (2.14) now reads

$$
\left(\frac{F(t)}{t}\right)^{r}-\left(\frac{F(s)}{s}\right)^{r} \leq\|a\|_{A_{1}}^{r} \int_{t}^{s} \tau^{-1} d \tau=\|a\|_{A_{1}}^{r} \log \frac{s}{t} .
$$

Estimate (2.16) now reads

$$
\left(\frac{F(s)}{s}\right)^{r} \leq \frac{1}{r}\|a\|_{A_{1}}^{r}+\frac{1}{r}\|a\|_{A_{0}}^{r} s^{-r\left(1+\sigma_{0}\right)}
$$


so that

$$
\begin{aligned}
\left(\frac{F(t)}{t}\right)^{r} & \leq \frac{1}{r}\|a\|_{A_{1}}^{r}+\frac{1}{r}\|a\|_{A_{0}}^{r} s^{-r\left(1+\sigma_{0}\right)}+\|a\|_{A_{1}}^{r} \log \frac{s}{t} \\
& =c\left(\|a\|_{A_{1}}^{r}\left(1+\log \frac{s}{t}\right)+\|a\|_{A_{0}}^{r} s^{-r\left(1+\sigma_{0}\right)}\right)
\end{aligned}
$$

Therefore

$$
\begin{aligned}
F^{r}(t) & \leq c t^{r} s^{-r\left(1+\sigma_{0}\right)}\left(\|a\|_{A_{1}}^{r} s^{r\left(1+\sigma_{0}\right)}\left(1+\log \frac{s}{t}\right)+\|a\|_{A_{0}}^{r}\right) \\
& \leq c t^{r} s^{-r\left(1+\sigma_{0}\right)} J^{r}\left(s^{1+\sigma_{0}}\left(1+\log \frac{s}{t}\right)^{1 / r}, a ; \bar{A}\right) .
\end{aligned}
$$

We set

$$
\tau=s\left(1+\log \frac{s}{t}\right)^{1 / r\left(1+\sigma_{0}\right)}
$$

Then $\tau \geq s$ and so

$$
\tau \leq s\left(1+\log \frac{\tau}{t}\right)^{1 / r\left(1+\sigma_{0}\right)}
$$

which implies

$$
\frac{1}{s} \leq \frac{\left(1+\log \frac{\tau}{t}\right)^{1 / r\left(1+\sigma_{0}\right)}}{\tau} .
$$

Moreover $s \mapsto s(1+\log (s / t))^{1 / r\left(1+\sigma_{0}\right)}$ maps $[t, \infty)$ onto itself and is one-toone. Thus for all $\tau \geq t$,

$$
\begin{aligned}
F(t) & \leq c t s^{-\left(1+\sigma_{0}\right)} J\left(s^{1+\sigma_{0}}\left(1+\log \frac{s}{t}\right)^{1 / r}, a ; \bar{A}\right) \\
& =c t s^{-\left(1+\sigma_{0}\right)} J\left(\tau^{1+\sigma_{0}}, a ; \bar{A}\right) \\
& \leq c t\left(1+\log \frac{\tau}{t}\right)^{1 / r} \tau^{-\left(1+\sigma_{0}\right)} J\left(\tau^{1+\sigma_{0}}, a ; \bar{A}\right) \\
& =c \frac{t}{\tau}\left(1+\log \frac{\tau}{t}\right)^{1 / r} \tau^{-\sigma_{0}} J\left(\tau^{1+\sigma_{0}}, a ; \bar{A}\right) \\
& =c \Psi^{1 / r}\left(\left(\frac{t}{\tau}\right)^{r}, \sigma_{0}, 0\right) \tau^{-\sigma_{0}} J\left(\tau^{1+\sigma_{0}}, a ; \bar{A}\right)
\end{aligned}
$$

Since $K \leq F$ we proved

$$
K(t, T a ; \bar{B}) \leq c \Psi^{1 / r}\left((t / s)^{r}, \sigma_{0}, \sigma_{1}\right) s^{-\sigma_{0}} J\left(s^{1+\sigma_{0}+\sigma_{1}}, a ; \bar{A}\right)
$$

for $t \leq s$ and $\sigma_{1} \geq 0$.

Applying the last inequality to the couples $\bar{A}^{T}$ and $\bar{B}^{T}$ and recalling that $T$ is a weak-type $\left(\sigma_{1}, \sigma_{0} ; r\right)$ operator with respect to these couples, we 
have, for $t \leq s$,

$$
K\left(t, T a ; \bar{B}^{T}\right) \leq c \Psi^{1 / r}\left((t / s)^{r}, \sigma_{1}, \sigma_{0}\right) s^{-\sigma_{1}} J\left(s^{1+\sigma_{0}+\sigma_{1}}, a ; \bar{A}^{T}\right)
$$

so that

$$
t K(1 / t, T a ; \bar{B}) \leq c \Psi^{1 / r}\left((t / s)^{r}, \sigma_{1}, \sigma_{0}\right) s^{-\sigma_{1}} s^{1+\sigma_{0}+\sigma_{1}} J\left(s^{-\left(1+\sigma_{0}+\sigma_{1}\right)}, a ; \bar{A}\right)
$$

and hence

$$
K(1 / t, T a ; \bar{B}) \leq c(t / s)^{-1} \Psi^{1 / r}\left((t / s)^{r}, \sigma_{1}, \sigma_{0}\right) s^{\sigma_{0}} J\left(s^{-\left(1+\sigma_{0}+\sigma_{1}\right)}, a ; \bar{A}\right) .
$$

Replacing $1 / t$ by $t$ and $1 / s$ by $s$, and using (2.10), we have

$$
\begin{aligned}
K(t, T a ; \bar{B}) & \leq c(t / s) \Psi^{1 / r}\left((s / t)^{r}, \sigma_{1}, \sigma_{0}\right) s^{-\sigma_{0}} J\left(s^{1+\sigma_{0}+\sigma_{1}}, a ; \bar{A}\right) \\
& =c \Psi^{1 / r}\left((t / s)^{r}, \sigma_{0}, \sigma_{1}\right) s^{-\sigma_{0}} J\left(s^{1+\sigma_{0}+\sigma_{1}}, a ; \bar{A}\right)
\end{aligned}
$$

also for $s \leq t$, and (2.13) is proved.

The following theorem is a variation on the Strong Fundamental Lemma of real interpolation theory (see [2], [3], [5]). We assume more on the decomposed element, $a \in A_{0} \cap A_{1}$ instead of $a \in A_{0}+A_{1}$. On the other hand, we do not have to assume that $\bar{A}$ is a Gagliardo couple.

TheOREM 2.10. Let $\bar{A}=\left(A_{0}, A_{1}\right)$ be an interpolation couple and let $a \in A_{0} \cap A_{1}$. Then for any $\varepsilon>0$ there is a finite decomposition

$$
a=u_{0}+\sum_{i=1}^{n} u_{i}+u_{\infty}
$$

and numbers $0<t_{0}<\varepsilon, t_{\infty}>1 / \varepsilon$ and $t_{1}, \ldots, t_{n}>0$ so that $u_{0}, u_{i}, u_{\infty} \in$ $A_{0} \cap A_{1}$ and for all $0<t, q<\infty$,

$$
\begin{aligned}
& \left(\sum_{i=1}^{n}\left(\min \left\{1, t / t_{i}\right\} J\left(t_{i}, u_{i} ; \bar{A}\right)\right)^{q}\right)^{1 / q} \leq c K(t, a ; \bar{A}), \\
& J\left(t_{0}, u_{0} ; \bar{A}\right) \leq c t_{0}\|a\|_{A_{1}}, \quad J\left(t_{\infty}, u_{\infty} ; \bar{A}\right) \leq c\|a\|_{A_{0}} .
\end{aligned}
$$

Here $c$ is a constant which depends only on $q, c_{0}, c_{1}$ where $c_{0}, c_{1}$ are the constants in the quasi-triangle inequalities for $A_{0}$ and $A_{1}$.

In order not to interrupt the proof of the main theorem we postpone the proof of Theorem 2.10 to Section 4 .

In the proof of the next lemma we shall use the concept of quasi-concavity.

Let us recall that a function $\psi: \mathbb{R}_{+} \rightarrow \mathbb{R}_{+}$is said to be quasi-concave if both $\psi \nearrow$ and $\psi(s) / s \searrow$. For any function $\phi: \mathbb{R}_{+} \rightarrow \mathbb{R}_{+}$, we denote by $\phi^{\nabla}$ its least quasi-concave majorant. It can be readily seen that

$$
\phi^{\nabla}(t)=\sup _{s>0}\{\min (1, t / s) \phi(s)\} .
$$


Also, recall [2, p. 291] that

$$
\phi^{\nabla} \leq \widehat{\phi} \leq 2 \phi^{\nabla} .
$$

Lemma 2.11. Let $\bar{X}=\left(X_{0}, X_{1}\right)$ be an interpolation couple. Assume that

$$
g: \mathbb{R}_{+} \times\left(X_{0}+X_{1}\right) \rightarrow \mathbb{R}_{+}
$$

is such that $\hat{g} \backsim K(\cdot, \cdot ; \bar{X})$ and that $0 \leq \theta_{0}<\theta_{1} \leq 1$. For $t>0$ and $x \in X_{0}+X_{1}$, define

$$
G\left(t, x, \theta_{0}, \theta_{1}\right)=t^{-\theta_{0} /\left(\theta_{1}-\theta_{0}\right)} g\left(t^{1 /\left(\theta_{1}-\theta_{0}\right)}, x\right)
$$

and $F=\widehat{G}$. Then

$$
F\left(t, x, \theta_{0}, \theta_{1}\right) \backsim K\left(t, x ; \bar{X}_{\theta_{0}, \infty}, \bar{X}_{\theta_{1}, \infty}\right) .
$$

If $\theta_{0}=0$ we replace $\bar{X}_{\theta_{0}, \infty}$ by $X_{0}$ and if $\theta_{1}=1$ we replace $\bar{X}_{\theta_{1}, \infty}$ by $X_{1}$.

Proof. From Holmstedt's formula,

$$
\begin{aligned}
K(t, & \left.x ; \bar{X}_{\theta_{0}, \infty}, \bar{X}_{\theta_{1}, \infty}\right) \\
& \sim \sup _{0<s \leq t^{1 /\left(\theta_{1}-\theta_{0}\right)}}\left\{s^{-\theta_{0}} K(s, x ; \bar{X})\right\}+t \sup _{s \geq t^{1 /\left(\theta_{1}-\theta_{0}\right)}}\left\{s^{-\theta_{1}} K(s, x ; \bar{X})\right\} \\
& \sim \sup _{s>0}\left\{\min (1, t / s) s^{-\theta_{0} /\left(\theta_{1}-\theta_{0}\right)} K\left(s^{1 /\left(\theta_{1}-\theta_{0}\right)}, x ; \bar{X}\right)\right\} \\
& \sim \sup _{s>0}\left\{\min (1, t / s) s^{-\theta_{0} /\left(\theta_{1}-\theta_{0}\right)} \widehat{g}\left(s^{1 /\left(\theta_{1}-\theta_{0}\right)}, x\right)\right\} .
\end{aligned}
$$

Since, by (2.19), $g^{\nabla} \backsim \widehat{g}$, we have

$$
\begin{aligned}
K(t, x ; & \left.\bar{X}_{\theta_{0}, \infty}, \bar{X}_{\theta_{1}, \infty}\right) \\
& \sim \sup _{s>0}\left\{\min (1, t / s) s^{-\theta_{0} /\left(\theta_{1}-\theta_{0}\right)} g^{\nabla}\left(s^{1 /\left(\theta_{1}-\theta_{0}\right)}, x\right)\right\} \\
& \sim \sup _{s>0}\left\{\min (1, t / s) s^{-\theta_{0} /\left(\theta_{1}-\theta_{0}\right)} \sup _{u>0} \min \left\{1, s^{1 /\left(\theta_{1}-\theta_{0}\right)} / u\right\} g(u, x)\right\} \\
& =\sup _{u>0} g(u, x) \sup _{s>0}\left\{\min (1, t / s) s^{-\theta_{0} /\left(\theta_{1}-\theta_{0}\right)} \min \left\{1, s^{1 /\left(\theta_{1}-\theta_{0}\right)} / u\right\}\right\} \\
& =\sup _{u>0} g(u, x)\left(\min \left\{s^{-\theta_{0} /\left(\theta_{1}-\theta_{0}\right)}, s^{\left(1-\theta_{0}\right) /\left(\theta_{1}-\theta_{0}\right)} / u\right\}\right)^{\nabla}(t) \\
& \sim \sup _{u>0} g(u, x)\left(\min \left\{s^{-\theta_{0} /\left(\theta_{1}-\theta_{0}\right)}, s^{\left(1-\theta_{0}\right) /\left(\theta_{1}-\theta_{0}\right)} / u\right\}\right) \frown(t) .
\end{aligned}
$$

But $\phi_{1}(s)=s^{-\theta_{0} /\left(\theta_{1}-\theta_{0}\right)}$ is a decreasing function and $\phi_{2}(s)=s^{1-\theta_{0} /\left(\theta_{1}-\theta_{0}\right)} / u$ is an increasing function so that

$$
\min \left\{\phi_{1}, \phi_{2}\right\}(s)= \begin{cases}\phi_{2}(s) & \text { if } 0<s<u^{\theta_{1}-\theta_{0}} \\ \phi_{1}(s) & \text { if } s \geq u^{\theta_{1}-\theta_{0}}\end{cases}
$$


Since $\phi_{2}$ is a convex function and $\phi_{1}$ is a decreasing function,

Therefore

$$
\left(\min \left\{\phi_{1}, \phi_{2}\right\}\right)^{-}(t)=u^{-\theta_{0}} \min \left\{1, \frac{t}{u^{\theta_{1}-\theta_{0}}}\right\} .
$$

$$
\begin{aligned}
K\left(t, x ; \bar{X}_{\theta_{0}, \infty}, \bar{X}_{\theta_{1}, \infty}\right) & \backsim \sup _{u>0} u^{-\theta_{0}} \min \left\{1, t / u^{\theta_{1}-\theta_{0}}\right\} g(u, x) \\
& =\sup _{s>0} s^{-\theta_{0} /\left(\theta_{1}-\theta_{0}\right)} \min \{1, t / s\} g\left(s^{1 /\left(\theta_{1}-\theta_{0}\right)}, x\right) \\
& =\left(s^{-\theta_{0} /\left(\theta_{1}-\theta_{0}\right)} g\left(s^{1 /\left(\theta_{1}-\theta_{0}\right)}, x\right)\right)^{\nabla}(t) \\
& \backsim\left(s^{-\theta_{0} /\left(\theta_{1}-\theta_{0}\right)} g\left(s^{1 /\left(\theta_{1}-\theta_{0}\right)}, x\right)\right)(t)=F\left(t, x, \theta_{0}, \theta_{1}\right) .
\end{aligned}
$$

When $\theta_{0}=0$, Holmstedt's formula implies

$$
\begin{aligned}
K\left(t, x ; X_{0}, \bar{X}_{\theta_{1}, \infty}\right) & \backsim t \sup _{t^{1 /\left(\theta_{1}-\theta_{0}\right)} \leq s}\left\{s^{-\theta_{1}} K(s, x ; \bar{X})\right\} \\
& \backsim \sup _{s>0}\left\{\min (1, t / s) K\left(s^{1 / \theta_{1}}, x ; \bar{X}\right)\right\} \\
& \sim \sup _{s>0}\left\{\min (1, t / s) \widehat{g}\left(s^{1 / \theta_{1}}, x\right)\right\}
\end{aligned}
$$

and the calculation above implies

$$
K\left(t, x ; X_{0}, \bar{X}_{\theta_{1}, \infty}\right) \backsim g\left(s^{1 / \theta_{1}}, x\right) \frown(t)=F\left(t, x, 0, \theta_{1}\right) .
$$

Similarly

$$
K\left(t, x ; \bar{X}_{\theta_{0}, \infty}, X_{1}\right) \backsim\left(g\left(s^{1 /\left(1-\theta_{0}\right)}, x\right) s^{-\theta_{0} /\left(1-\theta_{0}\right)}\right) \frown(t)=F\left(t, x, \theta_{0}, 1\right) .
$$

The proof of the following lemma is elementary.

Lemma 2.12. If $p_{1}<p_{2}$ and $f: \mathbb{R}_{+} \rightarrow \mathbb{R}_{+}$is a non-decreasing function so that $f(2 t) \backsim f(t)$ then

$$
S_{p_{2}} f \leq c S_{p_{1}} f .
$$

We will now prove Theorem 2.5.

Proof of Theorem 2.5. Set $p=\min \{\alpha, r\}$. Recall also that

$$
\theta_{0}=\frac{\sigma_{0}}{1+\sigma_{0}+\sigma_{1}} \quad \text { and } \quad \theta_{1}=\frac{1+\sigma_{0}}{1+\sigma_{0}+\sigma_{1}}
$$

so that

$$
\sigma_{0}=\frac{\theta_{0}}{\theta_{1}-\theta_{0}} \quad \text { and } \quad \sigma_{1}=\frac{1-\theta_{1}}{\theta_{1}-\theta_{0}} .
$$

Let $a \in A_{0} \cap A_{1}$ and $t>0$ be given. Choose $\varepsilon=\varepsilon(t, a)>0$ so that for all $0<t_{0}<\varepsilon$ and all $t_{\infty}>1 / \varepsilon$ we have

$$
\begin{array}{rl}
\left(1+\left|\log \frac{t}{t_{0}^{\theta_{1}-\theta_{0}}}\right|\right)^{1 / r} t_{0}^{1-\theta_{0}}\|a\|_{A_{1}}+t_{\infty}^{-\theta_{1}} & t\left(1+\left|\log \frac{t}{t_{\infty}^{\theta_{1}-\theta_{0}}}\right|\right)^{1 / r}\|a\|_{A_{0}} \\
& \leq S_{p} K\left(\cdot, a ; \bar{A}_{\theta_{0}, \infty}, \bar{A}_{\theta_{1}, \infty}\right)(t) .
\end{array}
$$


Using Theorem 2.10 with $\varepsilon$ as above, we decompose $a$ as

$$
a=u_{0}+\sum_{i=1}^{n} u_{i}+u_{\infty}
$$

For any such decomposition we have, from Lemma 2.4,

$$
K^{\alpha}(t ; T a ; \bar{B}) \leq 2 M\left(K^{\alpha}\left(t, T u_{0} ; \bar{B}\right)+\sum_{i=1}^{n} K^{\alpha}\left(t, T u_{i} ; \bar{B}\right)+K^{\alpha}\left(t, T u_{\infty} ; \bar{B}\right)\right) .
$$

By Lemma 2.9,

$$
K\left(t, T u_{i} ; \bar{B}\right) \leq c \Psi^{1 / r}\left((t / s)^{r}, \sigma_{0}, \sigma_{1}\right) s^{-\sigma_{0}} J\left(s^{1+\sigma_{0}+\sigma_{1}}, u_{i} ; \bar{A}\right) .
$$

Let us apply the last inequality to $s=t_{i}^{\theta_{1}-\theta_{0}}$ where $t_{i}$ is as in Theorem 2.10. Then by $(2.20)$, we have

$$
K\left(t, T u_{i} ; \bar{B}\right) \leq c \Psi^{1 / r}\left(\left(\frac{t}{t_{i}^{\theta_{1}-\theta_{0}}}\right)^{r}, \sigma_{0}, \sigma_{1}\right) t_{i}^{-\theta_{0}} J\left(t_{i}, u_{i} ; \bar{A}\right) .
$$

Let us consider first $K\left(t, T u_{0} ; \bar{B}\right)$, so

$$
K\left(t, T u_{0} ; \bar{B}\right) \leq c \Psi^{1 / r}\left(\left(\frac{t}{t_{0}^{\theta_{1}-\theta_{0}}}\right)^{r}, \sigma_{0}, \sigma_{1}\right) t_{0}^{-\theta_{0}} J\left(t_{0}, u_{0} ; \bar{A}\right) .
$$

From Theorem 2.10, $J\left(t_{0}, u_{0} ; \bar{A}\right) \leq c t_{0}\|a\|_{A_{1}}$, and from the definition of $\Psi$,

$$
\Psi^{1 / r}\left(x^{r}, \sigma_{0}, \sigma_{1}\right) \leq c \min \{1, x\}(1+|\log x|)^{1 / r}
$$

so that

$$
\begin{aligned}
K\left(t, T u_{0} ; \bar{B}\right) & \leq c\left(1+\left|\log \frac{t}{t_{0}^{\theta_{1}-\theta_{0}}}\right|\right)^{1 / r} t_{0}^{1-\theta_{0}}\|a\|_{A_{1}} \\
& \leq c S_{p} K\left(\cdot, a ; \bar{A}_{\theta_{0}, \infty}, \bar{A}_{\theta_{1}, \infty}\right)(t) .
\end{aligned}
$$

Let us now consider $K\left(t, T u_{\infty} ; \bar{B}\right)$, so

$$
K\left(t, T u_{\infty} ; \bar{B}\right) \leq c \Psi^{1 / r}\left(\left(\frac{t}{t_{\infty}^{\theta_{1}-\theta_{0}}}\right)^{r}, \sigma_{0}, \sigma_{1}\right) t_{\infty}^{-\theta_{0}} J\left(t_{\infty}, u_{\infty} ; \bar{A}\right) .
$$

From Theorem 2.10, $J\left(t_{\infty}, u_{\infty} ; \bar{A}\right) \leq c\|a\|_{A_{0}}$ so that

$$
\begin{aligned}
K\left(t, T u_{\infty} ; \bar{B}\right) & \leq c t_{\infty}^{-\theta_{1}} t\left(1+\left|\log \frac{t}{t_{\infty}^{\theta_{1}-\theta_{0}}}\right|\right)^{1 / r}\|a\|_{A_{0}} \\
& \leq c S_{p} K\left(\cdot, a ; \bar{A}_{\theta_{0}, \infty}, \bar{A}_{\theta_{1}, \infty}\right)(t) .
\end{aligned}
$$

To prove the theorem it will therefore suffice to show

$$
\sum_{i=1}^{n} K^{\alpha}\left(t, T u_{i} ; \bar{B}\right) \leq c S_{p}^{\alpha} K\left(\cdot, a ; \bar{A}_{\theta_{0}, \infty}, \bar{A}_{\theta_{1}, \infty}\right)(t)
$$


By Lemma 2.8,

$$
\Psi\left(x, \sigma_{0}, \sigma_{1}\right) \backsim S h\left(\cdot, \sigma_{0}, \sigma_{1}\right)(x)
$$

where

$$
h\left(s, \sigma_{0}, \sigma_{1}\right)=s^{-\sigma_{0}} \min \left\{1, s^{1+\sigma_{0}+\sigma_{1}}\right\} .
$$

By (2.11), since $p \leq r$,

$$
\Psi^{1 / r}\left(\frac{t^{r}}{t_{i}^{\left(\theta_{1}-\theta_{0}\right) r}}, \sigma_{0}, \sigma_{1}\right) \leq \Psi^{1 / p}\left(\frac{t^{p}}{t_{i}^{\left(\theta_{1}-\theta_{0}\right) p}}, \sigma_{0}, \sigma_{1}\right)
$$

so that

$$
\begin{aligned}
\Psi^{p / r}\left(\frac{t^{r}}{t_{i}^{\left(\theta_{1}-\theta_{0}\right) r}}, \sigma_{0}, \sigma_{1}\right) & \leq c S h\left(\cdot, \sigma_{0}, \sigma_{1}\right)\left(\frac{t^{p}}{t_{i}^{\left(\theta_{1}-\theta_{0}\right) p}}\right) \\
& =c \int_{0}^{\infty} \min \left\{1, \frac{t^{p}}{s t_{i}^{\left(\theta_{1}-\theta_{0}\right) p}}\right\} s^{-\sigma_{0}} \min \left\{1, s^{1+\sigma_{0}+\sigma_{1}}\right\} \frac{d s}{s} .
\end{aligned}
$$

Let $u=s^{1 / p} t_{i}^{\theta_{1}-\theta_{0}}$. Then

$$
\Psi^{p / r}\left(\frac{t^{r}}{t_{i}^{\left(\theta_{1}-\theta_{0}\right) r}}, \sigma_{0}, \sigma_{1}\right) \leq c t_{i}^{p \theta_{0}} \int_{0}^{\infty} \min \left\{1, \frac{t^{p}}{u^{p}}\right\} u^{-p \sigma_{0}} \min \left\{1, \frac{u^{p\left(1+\sigma_{0}+\sigma_{1}\right)}}{t_{i}^{p}}\right\} \frac{d u}{u} .
$$

By (2.21), and since $p \leq \alpha$,

$$
\begin{aligned}
& \left(\sum_{i=1}^{n} K^{\alpha}\left(t, T u_{i} ; \bar{B}\right)\right)^{p / \alpha} \leq \sum_{i=1}^{n} K^{p}\left(t, T u_{i} ; \bar{B}\right) \\
& \quad \leq c \sum_{i=1}^{n} \Psi^{p / r}\left(\left(\frac{t}{t_{i}^{\theta_{1}-\theta_{0}}}\right)^{r}, \sigma_{0}, \sigma_{1}\right) t_{i}^{-p \theta_{0}} J^{p}\left(t_{i}, u_{i} ; \bar{A}\right) \\
& \quad \leq c \sum_{i=1}^{n} J^{p}\left(t_{i}, u_{i} ; \bar{A}\right) \int_{0}^{\infty} \min \left\{1, \frac{t^{p}}{u^{p}}\right\} u^{-p \sigma_{0}} \min \left\{1, \frac{u^{p\left(1+\sigma_{0}+\sigma_{1}\right)}}{t_{i}^{p}}\right\} \frac{d u}{u} \\
& \quad=c \int_{0}^{\infty} \min \left\{1, \frac{t^{p}}{u^{p}}\right\} u^{-p \sigma_{0}}\left(\sum_{i=1}^{n}\left(\min \left\{1, \frac{u^{1+\sigma_{0}+\sigma_{1}}}{t_{i}}\right\} J\left(t_{i}, u_{i} ; \bar{A}\right)\right)^{p}\right) \frac{d u}{u}
\end{aligned}
$$

By Theorem 2.10,

$$
\sum_{i=1}^{n}\left(\min \left\{1, \frac{u^{1+\sigma_{0}+\sigma_{1}}}{t_{i}}\right\} J\left(t_{i}, u_{i} ; \bar{A}\right)\right)^{p} \leq c K^{p}\left(u^{1+\sigma_{0}+\sigma_{1}}, a ; \bar{A}\right)
$$

so that

$$
\left(\sum_{i=1}^{n} K^{\alpha}\left(t, T u_{i} ; \bar{B}\right)\right)^{p / \alpha} \leq c \int_{0}^{\infty} \min \left\{1, \frac{t^{p}}{u^{p}}\right\}\left[u^{-\sigma_{0}} K\left(u^{1+\sigma_{0}+\sigma_{1}}, a ; \bar{A}\right)\right]^{p} \frac{d u}{u} .
$$


By Lemma 2.11 with $g=K(\cdot, \cdot ; \bar{A})$,

$$
\left[u^{-\theta_{0} /\left(\theta_{1}-\theta_{0}\right)} K\left(u^{1 /\left(\theta_{1}-\theta_{0}\right)}, a ; \bar{A}\right)\right]^{\frown}(s) \backsim K\left(s, a ; \bar{A}_{\theta_{0}, \infty}, \bar{A}_{\theta_{1}, \infty}\right) .
$$

Since

$$
u^{-\sigma_{0}} K\left(u^{1+\sigma_{0}+\sigma_{1}}, a ; \bar{A}\right)=u^{-\theta_{0} /\left(\theta_{1}-\theta_{0}\right)} K\left(u^{1 /\left(\theta_{1}-\theta_{0}\right)}, a ; \bar{A}\right)
$$

(see $(2.20)$ ), and since, of course, $f \leq \widehat{f}$, we have

$$
\begin{aligned}
\left(\sum_{i=1}^{n} K^{\alpha}\left(t, T u_{i} ; \bar{B}\right)\right)^{p / \alpha} & \leq c \int_{0}^{\infty} \min \left\{1, \frac{t^{p}}{u^{p}}\right\} K^{p}\left(u, a ; \bar{A}_{\theta_{0}, \infty}, \bar{A}_{\theta_{1}, \infty}\right) \frac{d u}{u} \\
& =S_{p}^{p} K\left(\cdot, a ; \bar{A}_{\theta_{0}, \infty}, \bar{A}_{\theta_{1}, \infty}\right)(t) .
\end{aligned}
$$

If $\sigma_{0}, \sigma_{1}>0$ then $\Psi\left(x, \sigma_{0}, \sigma_{1}\right)=\min \{1, x\}$ so that instead of (2.22) we have

$$
\Psi^{1 / r}\left(\frac{t^{r}}{t_{i}^{\left(\theta_{1}-\theta_{0}\right) r}}, \sigma_{0}, \sigma_{1}\right)=\Psi^{1 / \alpha}\left(\frac{t^{\alpha}}{t_{i}^{\left(\theta_{1}-\theta_{0}\right) \alpha}}, \sigma_{0}, \sigma_{1}\right)
$$

and continue the proof with $\alpha$ instead of $p$, proving the stronger

$$
K(t, T a ; \bar{B}) \leq c M_{0} S_{\alpha}\left[K\left(s, a ; \bar{A}_{\theta_{0}, \infty}, \bar{A}_{\theta_{1}, \infty}\right)\right]\left(\frac{M_{1}}{M_{0}} t\right) .
$$

If $\sigma_{0}=0$ and $\sigma_{1}=0$ we can prove another version of Theorem 2.5. The new version is stronger than (2.7) for $r>\alpha$.

TheOREM 2.13. If $T: \bar{A} \rightarrow \bar{B}$ is a weak-type $(0,0 ; r)$ operator with norms $M_{0}, M_{1}$, then for any $a \in A_{0} \cap A_{1}, 0<\beta<r$, and $t>0$,

$$
K(t, T a ; \bar{B}) \leq c M_{0} K^{1-\beta / r}\left(\frac{M_{1}}{M_{0}} t, a ; \bar{A}\right)\left[S_{\beta} K(\cdot, a ; \bar{A})\left(\frac{M_{1}}{M_{0}} t\right)\right]^{\beta / r} .
$$

Proof. The proof is similar to that of Theorem 2.5. We again assume $M_{0}=M_{1}=1$, and given $a \in A_{0} \cap A_{1}$ and $t>0$ we choose $\varepsilon=\varepsilon(t, a)>0$ so that for all $0<t_{0}<\varepsilon$ and for all $t_{\infty}>1 / \varepsilon$ we have

$$
\begin{aligned}
\left(1+\left|\log \frac{t}{t_{0}}\right|\right)^{1 / r} t_{0}\|a\|_{A_{1}}+\frac{t}{t_{\infty}} & \left(1+\left|\log \frac{t}{t_{\infty}}\right|\right)^{1 / r}\|a\|_{A_{1}} \\
& \leq K^{1-\beta / r}(t, a ; \bar{A})\left[S_{\beta} K(\cdot, a ; \bar{A})(t)\right]^{\beta / r} .
\end{aligned}
$$

We apply Theorem 2.10 with $\varepsilon$ as above, getting $u_{i}$ and $t_{i}$ as in that theorem. Repeating the argument of the proof of Theorem 2.5 we see that it suffices to show that

$$
\left(\sum_{i=1}^{n} K^{\alpha}\left(t, T u_{i} ; \bar{B}\right)\right)^{1 / \alpha} \leq c K^{1-\beta / r}(t, a ; \bar{A})\left[S_{\beta} K(\cdot, a ; \bar{A})(t)\right]^{\beta / r} .
$$


By (2.13),

$$
\begin{aligned}
K\left(t, T u_{i} ; B\right) & \leq c \Psi^{1 / r}\left(\left(t / t_{i}\right)^{r}, 0,0\right) J\left(t_{i}, u_{i} ; \bar{A}\right) \\
& \leq c \min \left\{1, t / t_{i}\right\}\left(1+\left|\log \left(t / t_{i}\right)\right|\right)^{1 / r} J\left(t_{i}, u_{i} ; \bar{A}\right) .
\end{aligned}
$$

Let $0<\gamma<\min \{\alpha, r\}$. Then

$$
\begin{aligned}
\left(\sum_{i=1}^{n} K^{\alpha}\left(t, T u_{i} ; B\right)\right)^{\gamma / \alpha} & \leq \sum_{i=1}^{n} K^{\gamma}\left(t, T u_{i} ; B\right) \\
& \leq c \sum_{i=1}^{n}\left(\min \left\{1, t / t_{i}\right\}\left(1+\left|\log \left(t / t_{i}\right)\right|\right)^{1 / r} J\left(t_{i}, u_{i} ; \bar{A}\right)\right)^{\gamma} .
\end{aligned}
$$

For any $0<\beta<r$ we have

$$
\begin{aligned}
\min \left\{1, t / t_{i}\right\}(1+\mid & \left.\log \left(t / t_{i}\right) \mid\right)^{1 / r} J\left(t_{i}, u_{i} ; \bar{A}\right) \\
= & \left.\left(\min \left\{1, t / t_{i}\right\}\right)^{\beta}\left(1+\left|\log \left(t / t_{i}\right)\right|\right) J^{\beta}\left(t_{i}, u_{i} ; \bar{A}\right)\right)^{1 / r} \\
& \times\left(\min \left\{1, t / t_{i}\right\} J\left(t_{i}, u_{i} ; \bar{A}\right)\right)^{1-\beta / r} \\
\backsim & \left(\left(\min \left\{1, t / t_{i}\right\}\right)^{\beta}\left(1+\left|\log \left(t / t_{i}\right)^{\beta}\right|\right) J^{\beta}\left(t_{i}, u_{i} ; \bar{A}\right)\right)^{1 / r} \\
& \times\left(\min \left\{1, t / t_{i}\right\} J\left(t_{i}, u_{i} ; \bar{A}\right)\right)^{1-\beta / r} .
\end{aligned}
$$

In this case, by Hölder's inequality,

$$
\begin{aligned}
\sum_{i=1}^{n} K^{\gamma}\left(t, T u_{i} ; \bar{B}\right) \leq & c\left(\sum_{i=1}^{n} \min \left\{1,\left(t / t_{i}\right)^{\beta}\right\}\left(1+\left|\log \left(t / t_{i}\right)^{\beta}\right|\right) J^{\beta}\left(t_{i}, u_{i} ; \bar{A}\right)\right)^{\gamma / r} \\
& \times\left(\sum_{i=1}^{n}\left(\min \left\{1, t / t_{i}\right\} J\left(t_{i}, u_{i} ; \bar{A}\right)\right)^{\gamma \frac{r-\beta}{r-\gamma}}\right)^{\frac{r-\gamma}{r}} .
\end{aligned}
$$

By Lemma 2.8,

$$
\min \{1, t\}(1+|\log t|) \backsim S(\min \{1, \cdot\})(t)
$$

so that

$$
\begin{aligned}
& \sum_{i=1}^{n} \min \left\{1,\left(t / t_{i}\right)^{\beta}\right\}\left(1+\left|\log \left(t / t_{i}\right)^{\beta}\right|\right) J^{\beta}\left(t_{i}, u_{i} ; \bar{A}\right) \\
& \backsim \sum_{i=1}^{n} J^{\beta}\left(t_{i}, u_{i} ; \bar{A}\right) S(\min \{1, \cdot\})\left(\left(t / t_{i}\right)^{\beta}\right) \\
& \backsim \sum_{i=1}^{n} \int_{0}^{\infty} \min \left\{1,\left(\frac{t}{t_{i}}\right)^{\beta} \frac{1}{s}\right\} \min \{1, s\} J^{\beta}\left(t_{i}, u_{i} ; \bar{A}\right) \frac{d s}{s} \\
& \backsim \int_{0}^{\infty} \min \left\{1,(t / s)^{\beta}\right\}\left(\sum_{i=1}^{n}\left(\min \left\{1, s / t_{i}\right\} J\left(t_{i}, u_{i} ; \bar{A}\right)\right)^{\beta}\right) \frac{d s}{s} .
\end{aligned}
$$


By Theorem 2.10,

$$
\sum_{i=1}^{n}\left(\min \left\{1, s / t_{i}\right\} J\left(t_{i}, u_{i} ; \bar{A}\right)\right)^{\beta} \leq c K^{\beta}(s, a ; \bar{A})
$$

so that

$$
\begin{aligned}
& \sum_{i=1}^{n} \min \left\{1,\left(t / t_{i}\right)^{\beta}\right\}\left(1+\left|\log \left(t / t_{i}\right)^{\beta}\right|\right) J^{\beta}\left(t_{i}, u_{i} ; \bar{A}\right) \\
& \leq c \int_{0}^{\infty}(\min \{1, t / s\} K(s, a ; \bar{A}))^{\beta} \frac{d s}{s}=c S_{\beta}^{\beta} K(\cdot, a ; \bar{A})(t) .
\end{aligned}
$$

Again by Theorem 2.10,

$$
\sum_{i=1}^{n}\left(\min \left\{1, t / t_{i}\right\} J\left(t_{i}, u_{i} ; \bar{A}\right)\right)^{\gamma \frac{r-\beta}{r-\gamma}} \leq c K^{\gamma \frac{r-\beta}{r-\gamma}}(t, a ; \bar{A}) .
$$

Therefore

$$
\left(\sum_{i=1}^{n} K^{\alpha}\left(t, T u_{i} ; \bar{B}\right)\right)^{\gamma / \alpha} \leq c K^{(1-\beta / r) \gamma}(t, a ; \bar{A})\left[S_{\beta} K(\cdot, a ; \bar{A})(t)\right]^{\gamma \beta / r}
$$

and (2.24) is proved.

REMARK 2.14. A strong-type operator satisfies

$$
K(t, T a ; \bar{B}) \leq c M_{0} K\left(\frac{M_{1}}{M_{0}} t, a ; \bar{A}\right)
$$

so that the right-hand side of (2.23) represents an intermediate expression between (2.26) and the weaker (2.7). For a fixed $\beta$, the constant in (2.23) remains bounded as $r$ increases, and so $(2.23)$ is a better inequality when the $(0,0 ; r)$ weak-type holds for a larger $r$.

3. Another condition for weak-type operators. In [9] and [11] weak-type classes were defined using differences rather than derivatives. Weak-type operators are simply operators that are continuous on the corresponding weak-type classes. Let us see the connection between the definition using differences and the one using derivatives.

Lemma 3.1. Assume that $H: \mathbb{R}_{+} \rightarrow \mathbb{R}_{+}$is a concave function. Then for $r>0, \gamma \geq 0, \lambda>1$,

$$
\sup _{t>0}\left(t^{\gamma}\left(H^{r}(\lambda t)-H^{r}(t)\right)\right) \backsim \sup _{t>0}\left(t^{\gamma+1}\left(H^{r}\right)^{\prime}(t)\right) .
$$


Proof. Since $H: \mathbb{R}_{+} \rightarrow \mathbb{R}_{+}$is concave, we have $H \nearrow, H^{\prime} \searrow$, and $H(\lambda t) \leq \lambda H(t)$ and so

$$
\begin{aligned}
H^{r}(\lambda t)-H^{r}(t) & =\int_{t}^{\lambda t}\left(H^{r}\right)^{\prime}(s) d s=r \int_{t}^{\lambda t} H^{r-1}(s) H^{\prime}(s) d s \\
& \geq r(\lambda-1) t H^{r-1}(t) H^{\prime}(\lambda t) \geq \frac{r(\lambda-1)}{\lambda^{r-1}} t H^{r-1}(\lambda t) H^{\prime}(\lambda t) \\
& =\frac{\lambda-1}{\lambda^{r}}(\lambda t)\left(H^{r}\right)^{\prime}(\lambda t)
\end{aligned}
$$

so that

$$
\begin{aligned}
& \sup _{t>0}\left(t^{\gamma}\left(H^{r}(\lambda t)-H^{r}(t)\right)\right) \geq \sup _{t>0}\left(t^{\gamma} \frac{\lambda-1}{\lambda^{r}}(\lambda t)\left(H^{r}\right)^{\prime}(\lambda t)\right) \\
& =\frac{\lambda-1}{\lambda^{r+\gamma}} \sup _{t>0}\left((\lambda t)^{\gamma+1}\left(H^{r}\right)^{\prime}(\lambda t)\right)=\frac{\lambda-1}{\lambda^{r+\gamma}} \sup _{t>0}\left(t^{\gamma+1}\left(H^{r}\right)^{\prime}(t)\right) .
\end{aligned}
$$

The opposite inequality is trivial.

Lemma 3.2. Suppose that $H: \mathbb{R}_{+} \rightarrow \mathbb{R}_{+}$has a finite concave majorant and satisfies for some $\lambda>1, r>0, \gamma, A \geq 0$ and all $t>0$,

$$
H^{r}(\lambda t)-H^{r}(t) \leq A t^{-\gamma} \text {. }
$$

Then for all $t>0$,

$$
\widehat{H}^{r}(\lambda t)-\widehat{H}^{r}(t) \leq C A t^{-\gamma}
$$

where $C=C(\lambda, \gamma, r)$.

Proof. Consider first the case $\gamma>0$. Iterating (3.1) we obtain, for all $n \geq 1$ and $x>0$,

$$
H^{r}\left(\lambda^{n} x\right) \leq H^{r}(x)+\left(\sum_{k=0}^{n-1} \lambda^{-\gamma k}\right) A x^{-\gamma} \leq H^{r}(x)+C A x^{-\gamma}
$$

where

$$
C=\sum_{k=0}^{\infty} \lambda^{-\gamma k}
$$

Write $\lambda^{n} x=u$ so that

$$
H^{r}(u) \leq H^{r}\left(\lambda^{-n} u\right)+C A u^{-\gamma} \lambda^{-n \gamma} \leq \widehat{H}^{r}\left(\lambda^{-n} u\right)+C A u^{-\gamma} \lambda^{n \gamma} .
$$

By [7, Theorem 12.2] the least concave majorant of $\phi: \mathbb{R}_{+} \rightarrow \mathbb{R}_{+}$is given by

$$
\widehat{\phi}(t)=\inf _{s>0} \sup _{u>0}(\phi(u)+s(t-u))
$$

so that

$$
\widehat{H}(\lambda t)=\inf _{s>0} \sup _{u>0}(H(u)+s(\lambda t-u)) .
$$


Let us estimate $\sup _{u>0}(H(u)+s(\lambda t-u))$. It will be convenient to define

$$
U(t)=\left(\widehat{H}^{r}(t)+C \lambda^{\gamma} A t^{-\gamma}\right)^{1 / r} .
$$

For $0<u \leq t$,

$$
\begin{aligned}
H(u)+s(\lambda t-u) & \leq \widehat{H}(u)+s(\lambda t-u) \leq \widehat{H}(t)+s(\lambda t-u) \\
& \leq \widehat{H}(t)+s \lambda t \leq U(t)+s \lambda t .
\end{aligned}
$$

For $\lambda^{n-1} t<u \leq \lambda^{n} t$, by (3.3) we obtain

$$
\begin{aligned}
H(u) & \leq\left(\widehat{H}^{r}\left(\lambda^{-n} u\right)+C A u^{-\gamma} \lambda^{n \gamma}\right)^{1 / r} \leq\left(\widehat{H}^{r}(t)+C A \lambda^{-(n-1) \gamma} t^{-\gamma} \lambda^{n \gamma}\right)^{1 / r} \\
& =\left(\widehat{H}^{r}(t)+C \lambda^{\gamma} A t^{-\gamma}\right)^{1 / r}=U(t) .
\end{aligned}
$$

Thus

$$
\begin{aligned}
\sup _{\lambda^{n-1} t<u \leq \lambda^{n} t}(H(u)+s(\lambda t-u)) & \leq \sup _{\lambda^{n-1} t<u \leq \lambda^{n} t}(U(t)+s(\lambda t-u)) \\
& =U(t)+s\left(\lambda t-\lambda^{n-1} t\right) \leq U(t)+s \lambda t .
\end{aligned}
$$

Therefore

$$
\begin{aligned}
& \sup _{u>0}(H(u)+s(\lambda t-u)) \\
& \quad \max \left(\sup _{0<u \leq t}(H(u)+s(\lambda t-u)), \sup _{n \geq 1} \sup _{\lambda^{n-1} t<u \leq \lambda^{n} t}(H(u)+s(\lambda t-u))\right) \\
& \quad \leq U(t)+s \lambda t
\end{aligned}
$$

so that

$$
\begin{aligned}
\hat{H}(\lambda t) & =\inf _{s>0} \sup _{u>0}(H(u)+s(\lambda t-u)) \\
& \leq \inf _{s>0}(U(t)+s \lambda t)=U(t)=\left(\widehat{H}^{r}(t)+C \lambda^{\gamma} A t^{-\gamma}\right)^{1 / r}
\end{aligned}
$$

and we have proved the lemma in the case $\gamma>0$.

If $\gamma=0$ condition (3.1) reads

$$
H^{r}(\lambda t) \leq H^{r}(t)+A \text {. }
$$

The proof that $\widehat{H}$ also satisfies (3.4) when $r \leq 1$ is easy. We have

$$
H^{r}(\lambda t) \leq \hat{H}^{r}(t)+A
$$

and since for $r \leq 1$ the function $\phi(s)=\left(A+s^{r}\right)^{1 / r}$ is concave so is

$$
\phi \circ \widehat{H}=\left(\widehat{H}^{r}(t)+A\right)^{1 / r} .
$$

It is easy to check that $(H(\lambda s)) \frown(t)=\widehat{H}(\lambda t)$ and so

$$
\widehat{H}(\lambda t) \leq\left(\widehat{H}^{r}(t)+A\right)^{1 / r} .
$$


We consider the case $r>1$. Clearly if (3.4) holds for $H$ it also holds for its least non-decreasing majorant:

$$
H^{\nearrow}(t)=\sup _{0<s \leq t} H(s) .
$$

Since also $\left(H^{\nearrow}\right)^{\frown}=\widehat{H}$, without loss of generality we assume that $H$ itself is non-decreasing.

Let us see next that if $H$ is non-decreasing and satisfies (3.4) then so does its least quasi-concave majorant, $H^{\nabla}$ (see (2.18)). Iterating (3.4) we have, for $n \geq 1$,

$$
H^{r}\left(\lambda^{n} t\right) \leq H^{r}(t)+n A
$$

For $\lambda^{n-1} t \leq s \leq \lambda^{n} t$ we have

$$
H^{r}(s) \leq H^{r}\left(\lambda^{n} t\right) \leq H^{r}(t)+n A
$$

so that for $s \geq t$,

$$
H^{r}(s) \leq H^{r}(t)+\left(1+\log _{\lambda} \frac{s}{t}\right) A
$$

Since

$$
H^{\nabla}(t)=\max \left\{\sup _{0<s \leq t} H(s), \sup _{s \geq t} \frac{t}{s} H(s)\right\},
$$

for $H$ non-decreasing we have

$$
H^{\nabla}(t)=\max \left\{H(t), \sup _{s \geq t} \frac{t}{s} H(s)\right\}=\sup _{s \geq t} \frac{t}{s} H(s) .
$$

Therefore

$$
H^{\nabla}(\lambda t)=\sup _{s \geq \lambda t} \frac{\lambda t}{s} H(s)=\sup _{s \geq t} \frac{t}{s} H(\lambda s) .
$$

From (3.5),

$$
\begin{aligned}
H^{\nabla}(\lambda t) & =\sup _{s \geq t} \frac{t}{s} H(\lambda s) \leq \sup _{s \geq t} \frac{t}{s}\left(H^{r}(t)+\left(1+\log _{\lambda} \frac{\lambda s}{t}\right) A\right)^{1 / r} \\
& =\sup _{s \geq t}\left(\left(\frac{t}{s}\right)^{r} H^{r}(t)+\left(\frac{t}{s}\right)^{r}\left(2+\log _{\lambda} \frac{s}{t}\right) A\right)^{1 / r} \\
& \leq \sup _{s \geq t}\left(H^{r}(t)+\left(\frac{t}{s}\right)^{r}\left(2+\log _{\lambda} \frac{s}{t}\right) A\right)^{1 / r} \\
& =\left(H^{r}(t)+\sup _{x \geq 1}\left(x^{-r}\left(2+\log _{\lambda} x\right) A\right)\right)^{1 / r}
\end{aligned}
$$

Set

$$
C=\sup _{x \geq 1}\left(x^{-r}\left(2+\log _{\lambda} x\right)\right)
$$


so that

$$
H^{\nabla}(\lambda t) \leq\left(H^{r}(t)+C A\right)^{1 / r} \leq\left(\left(H^{\nabla}(t)\right)^{r}+C A\right)^{1 / r} .
$$

Since

$$
\widehat{H} \leq\left(H^{\nabla}\right)^{\frown} \leq(\widehat{H})^{\frown}=\widehat{H}
$$

we have $\left(H^{\nabla}\right) \frown=\widehat{H}$ and so we can assume that $H$ is quasi-concave.

Since $r>1$, by (3.4), for any $x>0$,

$$
A \geq H^{r}(\lambda x)-H^{r}(x) \geq r H^{r-1}(x)(H(\lambda x)-H(x))
$$

so that

$$
H(\lambda x) \leq H(x)+\frac{A}{r H^{r-1}(x)} .
$$

Iterating the last inequality gives

$$
H\left(\lambda^{n} x\right) \leq \frac{A}{r} \sum_{k=0}^{n-1} \frac{1}{H^{r-1}\left(\lambda^{k} x\right)}+H(x)
$$

and since $H$ is a non-decreasing function,

$$
H\left(\lambda^{n} x\right) \leq \frac{A}{r} \frac{n}{H^{r-1}(x)}+H(x) .
$$

Thus, for $\lambda^{n-1} t \leq s \leq \lambda^{n} t$,

$$
\begin{aligned}
H(s) & \leq H\left(\lambda^{n} t\right) \leq \frac{A}{r} \frac{n}{H^{r-1}(t)}+H(t) \leq \frac{A}{r} \frac{1}{H^{r-1}(t)}\left(1+\log _{\lambda} \frac{s}{t}\right)+H(t) \\
& \leq \frac{A}{r} \frac{1}{H^{r-1}(t)}\left(1+\log _{\lambda} \frac{s}{t}\right)+\hat{H}(t)
\end{aligned}
$$

so that for all $s>0$,

$$
H(s) \leq \frac{A}{r} \frac{1}{H^{r-1}(t)}\left(1+\log _{\lambda}\left(1+\frac{s}{t}\right)\right)+\hat{H}(t) .
$$

The function (of $s$ ) on the right-hand side is concave and so

$$
\widehat{H}(s) \leq \frac{A}{r} \frac{1}{H^{r-1}(t)}\left(1+\log _{\lambda}\left(1+\frac{s}{t}\right)\right)+\widehat{H}(t) .
$$

Taking $s=\lambda t$ we have

$$
(\widehat{H}(\lambda t)-\widehat{H}(t)) H^{r-1}(t) \leq \frac{A}{r}\left(1+\log _{\lambda}(1+\lambda)\right) .
$$

Since $H$ is quasi-concave, by (2.19), $H \leq \widehat{H} \leq 2 H$ and so

$$
(\widehat{H}(\lambda t)-\widehat{H}(t))(\widehat{H})^{r-1}(t) \leq 2^{r-1} \frac{A}{r}\left(1+\log _{\lambda}(1+\lambda)\right) \text {. }
$$


But, since $\widehat{H}(\lambda t) \leq \lambda \widehat{H}(t)$,

$$
(\widehat{H}(\lambda t)-\widehat{H}(t))(\widehat{H})^{r-1}(\lambda t) \leq(2 \lambda)^{r-1} \frac{A}{r}\left(1+\log _{\lambda}(1+\lambda)\right) .
$$

Finally,

$$
\begin{aligned}
\widehat{H}^{r}(\lambda t)-\widehat{H}^{r}(t) & \leq r(\widehat{H}(\lambda t)-\widehat{H}(t))(\widehat{H})^{r-1}(\lambda t) \\
& \leq(2 \lambda)^{r-1} A\left(1+\log _{\lambda}(1+\lambda)\right)
\end{aligned}
$$

proving (3.2).

TheOREm 3.3. A K-subadditive operator $T: \bar{A} \rightarrow \bar{B}$ is a weak-type $\left(\sigma_{0}, \sigma_{1} ; r\right)$ operator iff there exists a function

$$
G: \mathbb{R}_{+} \times\left(B_{0}+B_{1}\right) \rightarrow \mathbb{R}_{+}
$$

so that $K(\cdot, \cdot ; \bar{B}) \leq G$ and for some $0<M_{0}, M_{1}<\infty$ and all $a \in A_{0} \cap A_{1}$,

$$
\sup _{t>0}\left(t^{\sigma_{0}}\left[G^{r}(\lambda t, T a)-G^{r}(t, T a)\right]_{+}^{1 / r}\right) \leq M_{0}\|a\|_{A_{0}}
$$

and

$$
\sup _{t>0}\left(t^{\sigma_{1}}\left[G_{T}^{r}(\lambda t, T a)-G_{T}^{r}(t, T a)\right]_{+}^{1 / r}\right) \leq M_{1}\|a\|_{A_{1}} .
$$

Proof. If $T$ is a weak-type $\left(\sigma_{0}, \sigma_{1} ; r\right)$ operator then there exists a function

$$
F: \mathbb{R}_{+} \times\left(B_{0}+B_{1}\right) \rightarrow \mathbb{R}_{+}
$$

concave in the first variable so that $K(\cdot, \cdot ; \bar{B}) \leq F$ and satisfying (2.1) and (2.2). From Lemma 3.1 it follows that $F$ satisfies (3.6) and (3.7).

Conversely, if $G$ satisfies (3.6) and (3.7) then, by Lemma 3.2, so does $F:=\widehat{G}$. Again from Lemma 3.1 it follows that $F$ satisfies (2.1) and (2.2). Of course, since $K \leq G$ then also $K \leq F$ and so we see that $T$ is a weak-type $\left(\sigma_{0}, \sigma_{1} ; r\right)$ operator.

Let us see that we need the Calderón operator in the statement of Theorem 2.5. Write

$$
\overline{L^{\infty}}:=\left(L^{\infty}\left(\mathbb{R}_{+}, d s\right), L_{s^{-1}}^{\infty}\left(\mathbb{R}_{+}, d s\right)\right) .
$$

The following theorem is proved in [2, p. 298].

Theorem 3.4. For all $f \in L^{\infty}\left(\mathbb{R}_{+}, d s\right)+L_{s^{-1}}^{\infty}\left(\mathbb{R}_{+}, d s\right)$,

$$
K\left(t, f ; \overline{L^{\infty}}\right)=|f|^{\frown}(t) .
$$

Let $f: \mathbb{R}_{+} \rightarrow \mathbb{R}_{+}, \sigma_{0}, \sigma_{1} \geq 0$ and $r>0$. We define

$$
T f(t)=S_{r}\left(u^{-\sigma_{0}}\left|f\left(u^{1+\sigma_{0}+\sigma_{1}}\right)\right|\right)(t) .
$$

TheOrem 3.5. T is a $K$-subadditive $\overline{L^{\infty}} \rightarrow \overline{L^{\infty}}$ weak-type $\left(\sigma_{0}, \sigma_{1} ; r\right)$ operator. 
Proof. It is easy to verify that $T$ satisfies (1.2) so that $T$ is $K$-subadditive. By (3.8),

$$
K\left(t, T f ; \overline{L^{\infty}}\right)=(T f) \frown(t) .
$$

For any non-negative function $g, S_{r} g$ is a quasi-concave function so that

$$
K\left(t, T f ; \overline{L^{\infty}}\right)=(T f)^{\frown} \backsim(T f)^{\nabla}=T f .
$$

By Theorem 3.3 if we prove (3.6) and (3.7) with $G=T f$ it will follow that $T$ is an $\overline{L^{\infty}} \rightarrow \overline{L^{\infty}}$ weak-type $\left(\sigma_{0}, \sigma_{1} ; r\right)$ operator. Now

$$
\begin{aligned}
G^{r}(\lambda t, T f) & -G^{r}(t, T f)=(T f)^{r}(\lambda t)-(T f)^{r}(t) \\
= & S_{r}^{r}\left(u^{-\sigma_{0}}\left|f\left(u^{1+\sigma_{0}+\sigma_{1}}\right)\right|\right)(\lambda t)-S_{r}^{r}\left(u^{-\sigma_{0}}\left|f\left(u^{1+\sigma_{0}+\sigma_{1}}\right)\right|\right)(t) \\
= & \int_{0}^{\infty}\left(\min \left\{1,\left(\frac{\lambda t}{s}\right)^{r}\right\}-\min \left\{1,\left(\frac{t}{s}\right)^{r}\right\}\right) s^{-\sigma_{0} r}\left|f\left(s^{1+\sigma_{0}+\sigma_{1}}\right)\right|^{r} \frac{d s}{s} .
\end{aligned}
$$

When $s \in(0, t)$,

$$
\min \left\{1,(\lambda t / s)^{r}\right\}=\min \left\{1,(t / s)^{r}\right\}=1
$$

so that

$$
\begin{aligned}
G^{r}(\lambda t, T f) & -G^{r}(t, T f) \\
& =\int_{t}^{\infty}\left(\min \left\{1,\left(\frac{\lambda t}{s}\right)^{r}\right\}-\left(\frac{t}{s}\right)^{r}\right) s^{-\sigma_{0} r}\left|f\left(s^{1+\sigma_{0}+\sigma_{1}}\right)\right|^{r} \frac{d s}{s} \\
& \leq \int_{t}^{\infty}\left(\left(\frac{\lambda t}{s}\right)^{r}-\left(\frac{t}{s}\right)^{r}\right) s^{-\sigma_{0} r}\left|f\left(s^{1+\sigma_{0}+\sigma_{1}}\right)\right|^{r} \frac{d s}{s} \\
& \leq\left(\lambda^{r}-1\right) t^{r}\|f\|_{L^{\infty}}^{r} \int_{t}^{\infty} s^{-r} s^{-\sigma_{0} r} \frac{d s}{s}=c\|f\|_{L^{\infty}}^{r} t^{-\sigma_{0} r}
\end{aligned}
$$

and we showed that (3.6) holds.

Let us verify (3.7). We have

$$
\begin{aligned}
& G_{T}^{r}(\lambda t, T f)-G_{T}^{r}(t, T f)=\lambda^{r} t^{r}(T f)^{r}\left(\frac{1}{\lambda t}\right)-t^{r}(T f)^{r}\left(\frac{1}{t}\right) \\
& =\int_{0}^{\infty}\left(\lambda^{r} t^{r} \min \left\{1,\left(\frac{1}{\lambda t s}\right)^{r}\right\}-t^{r} \min \left\{1,\left(\frac{1}{t s}\right)^{r}\right\}\right) s^{-\sigma_{0} r}\left|f\left(s^{1+\sigma_{0}+\sigma_{1}}\right)\right|^{r} \frac{d s}{s} \\
& =\int_{0}^{\infty}\left(\min \left\{\lambda^{r} t^{r}, s^{-r}\right\}-\min \left\{t^{r}, s^{-r}\right\}\right) s^{-\sigma_{0} r}\left|f\left(s^{1+\sigma_{0}+\sigma_{1}}\right)\right|^{r} \frac{d s}{s} .
\end{aligned}
$$

When $s \in\left(t^{-1}, \infty\right)$,

$$
\min \left\{\lambda^{r} t^{r}, s^{-r}\right\}=\min \left\{t^{r}, s^{-r}\right\}
$$


so that

$$
\begin{aligned}
G_{T}^{r}(\lambda t, T f)-G_{T}^{r}(t, T f) & =\int_{0}^{1 / t}\left(\min \left\{\lambda^{r} t^{r}, s^{-r}\right\}-t^{r}\right) s^{-\sigma_{0} r}\left|f\left(s^{1+\sigma_{0}+\sigma_{1}}\right)\right|^{r} \frac{d s}{s} \\
& \leq \int_{0}^{1 / t}\left(\lambda^{r} t^{r}-t^{r}\right) s^{-\sigma_{0} r}\left|f\left(s^{1+\sigma_{0}+\sigma_{1}}\right)\right|^{r} \frac{d s}{s} \\
& \leq\left(\lambda^{r}-1\right) t^{r}\|f\|_{L_{s}^{\infty}}^{r} \int_{0}^{1 / t} s^{\left(1+\sigma_{0}+\sigma_{1}\right) r} s^{-\sigma_{0} r} \frac{d s}{s} \\
& =c\|f\|_{L_{s}^{\infty}}^{r} t^{-r \sigma_{1}}
\end{aligned}
$$

and we showed that (3.7) holds.

Let us see that if $g$ is a quasi-concave function and

$$
f(t)=t^{\frac{\sigma_{0}}{1+\sigma_{0}+\sigma_{1}}} g\left(t^{\frac{1}{1+\sigma_{0}+\sigma_{1}}}\right)
$$

then for all $r>0$ we have

$$
K\left(t, T f ; \overline{L^{\infty}}\right) \backsim S_{r}\left[K\left(\cdot, f ; \bar{L}_{\theta_{0}, \infty},{\overline{L^{\infty}}}_{\theta_{1}, \infty}\right)\right](t)
$$

where $\theta_{0}=\frac{\sigma_{0}}{1+\sigma_{0}+\sigma_{1}}$ and $\theta_{1}=\frac{1+\sigma_{0}}{1+\sigma_{0}+\sigma_{1}}$. Clearly

$$
g(s)=s^{-\theta_{0} /\left(\theta_{1}-\theta_{0}\right)} f\left(s^{1 /\left(\theta_{1}-\theta_{0}\right)}\right)=s^{-\sigma_{0}} f\left(s^{1+\sigma_{0}+\sigma_{1}}\right) .
$$

By $(3.8), K\left(t, f ; \overline{L^{\infty}}\right)=\widehat{f}(t)$. By Lemma 2.11,

$$
K\left(t, f ; \bar{L}_{\theta_{0}, \infty},{\overline{L^{\infty}}}_{\theta_{1}, \infty}\right) \backsim\left(s^{-\theta_{0} /\left(\theta_{1}-\theta_{0}\right)} f\left(s^{1 /\left(\theta_{1}-\theta_{0}\right)}\right)\right) \frown(t)=\widehat{g}(t) \backsim g(t) .
$$

By (3.9),

$$
K\left(t, T f ; \overline{L^{\infty}}\right) \backsim T f(t)=S_{r}\left(s^{-\sigma_{0}} f\left(s^{1+\sigma_{0}+\sigma_{1}}\right)\right)(t)=S_{r} g(t)
$$

so that

$$
K\left(t, T f ; \overline{L^{\infty}}\right) \backsim S_{r} g(t) \backsim S_{r}\left(K\left(t, f ;{\overline{L^{\infty}}}_{\theta_{0}, \infty},{\overline{L^{\infty}}}_{\theta_{1}, \infty}\right)\right)(t) .
$$

Thus, when $r \leq 1$, we have equivalence in (2.7).

4. Proof of the strong fundamental lemma. We present a proof of Theorem 2.10. The proof is a modification of those in [2], [3], and [5].

Proof of Theorem 2.10. For all $s>0$ let $a_{0}(s)$ be such that

$$
\left\|a_{0}(s)\right\|_{A_{0}}+s\left\|a-a_{0}(s)\right\|_{A_{1}} \leq 2 K(s, a ; \bar{A}) .
$$

Therefore

$$
\left\|a_{0}(s)\right\|_{A_{0}} \leq 2 K(s, a ; \bar{A}) \leq 2 s\|a\|_{A_{1}} .
$$


Also

$$
\begin{aligned}
s\left\|a_{0}(s)\right\|_{A_{1}} & =s\left\|a-\left(a-a_{0}(s)\right)\right\|_{A_{1}} \leq c_{1} s\|a\|_{A_{1}}+c_{1} s\left\|a-a_{0}(s)\right\|_{A_{1}} \\
& \leq c_{1} s\|a\|_{A_{1}}+2 c_{1} K(s, a ; \bar{A}) \leq 3 c_{1} s\|a\|_{A_{1}} .
\end{aligned}
$$

We therefore have

$$
J\left(s, a_{0}(s) ; \bar{A}\right)=\max \left\{\left\|a_{0}(s)\right\|_{A_{0}}, s\left\|a_{0}(s)\right\|_{A_{1}}\right\} \leq 3 c_{1} s\|a\|_{A_{1}} .
$$

Since

$\left\|a-a_{0}(s)\right\|_{A_{0}} \leq c_{0}\|a\|_{A_{0}}+c_{0}\left\|a_{0}(s)\right\|_{A_{0}} \leq c_{0}\|a\|_{A_{0}}+2 c_{0} K(s, a ; \bar{A}) \leq 3 c_{0}\|a\|_{A_{0}}$ and

$$
s\left\|a-a_{0}(s)\right\|_{A_{1}} \leq 2 K(s, a ; \bar{A}) \leq 2\|a\|_{A_{0}}
$$

we have

$$
J\left(s, a-a_{0}(s) ; \bar{A}\right) \leq 3 c_{0}\|a\|_{A_{0}} .
$$

Let $\tau_{0}<\varepsilon$ and $u_{0}=a_{0}\left(\tau_{0}\right)$. Then, by $(4.2)$,

$$
J\left(\tau_{0}, u_{0} ; \bar{A}\right) \leq 3 c_{1} \tau_{0}\|a\|_{A_{1}} .
$$

Assume that $u_{0}, u_{1}, \ldots, u_{i-1}$ and $\tau_{0}, \ldots, \tau_{i-1}$ have been defined. To construct $u_{i}$ and $\tau_{i}$ we define

$$
\begin{aligned}
& \tau_{i}^{+}=\sup \left\{s: K(s, a ; \bar{A}) \leq 2 K\left(\tau_{i-1}, a ; \bar{A}\right)\right\}, \\
& \tau_{i}^{-}=\max \left\{s: \frac{K(s, a ; \bar{A})}{s} \geq \frac{K\left(\tau_{i-1}, a ; \bar{A}\right)}{2 \tau_{i-1}}\right\}
\end{aligned}
$$

Since $a \in A_{0} \cap A_{1}$, we have

$$
\lim _{s \rightarrow \infty} \frac{K(s, a ; \bar{A})}{s} \leq \lim _{s \rightarrow \infty} \frac{\|a\|_{A_{0}}}{s}=0
$$

so that $\tau_{i}^{-}<\infty$.

It might happen that $\tau_{i}^{+}=\infty$. If $\tau_{i}^{+}<\infty$, we set $\tau_{i}=\max \left\{\tau_{i}^{+}, \tau_{i}^{-}\right\}$.

By the continuity of $K$, if $\tau_{i}^{+}<\infty$ we have

$$
K\left(\tau_{i}^{+}, a ; \bar{A}\right)=2 K\left(\tau_{i-1}, a ; \bar{A}\right) .
$$

If $\tau_{i}^{+}=\infty$, we take any $\tau_{i}>\max \left\{\tau_{i}^{-}, 1 / \varepsilon\right\}$ and stop.

Define in both cases

$$
u_{i}=a_{0}\left(\tau_{i}\right)-a_{0}\left(\tau_{i-1}\right) .
$$

Let us see that the sequence we defined is finite. For all $i$ we have

$$
K\left(\tau_{i}, a ; \bar{A}\right) \geq K\left(\tau_{i}^{+}, a ; \bar{A}\right)=2 K\left(\tau_{i-1}, a ; \bar{A}\right)
$$

so that

$$
K\left(\tau_{i}^{+}, a ; \bar{A}\right) \geq 2^{i} K\left(\tau_{0}, a ; \bar{A}\right)
$$


But since $a \in A_{0} \cap A_{1}$, we have $K(\cdot, a ; \bar{A}) \leq\|a\|_{A_{0}}<\infty$ and so $\tau_{i}^{+}=\infty$ for some $i$, that is, for all $s>\tau_{i-1}$,

$$
K(s, a ; \bar{A}) \leq 2 K\left(\tau_{i-1}, a ; \bar{A}\right) .
$$

We denote the last index by $n$, that is, $\tau_{n}^{+}=\infty$. We define

$$
u_{\infty}=a-a_{0}\left(\tau_{n}\right) \text {. }
$$

We have

$$
u_{0}+\sum_{i=1}^{n} u_{i}+u_{\infty}=a_{0}\left(\tau_{0}\right)+\sum_{i=1}^{n}\left(a_{0}\left(\tau_{i}\right)-a_{0}\left(\tau_{i-1}\right)\right)+\left(a-a_{0}\left(\tau_{n}\right)\right)=a .
$$

For $i \leq n-1$, that is, when $\tau_{i}^{+}<\infty$, we have (4.6) and similarly

$$
\frac{K\left(\tau_{i+1}, a ; \bar{A}\right)}{\tau_{i+1}} \leq \frac{K\left(\tau_{i+1}^{-}, a ; \bar{A}\right)}{\tau_{i+1}^{-}}=\frac{K\left(\tau_{i}, a ; \bar{A}\right)}{2 \tau_{i}} .
$$

From (4.3) it follows that

$$
J\left(\tau_{n}, u_{\infty} ; \bar{A}\right)=J\left(\tau_{n}, a-a_{0}\left(\tau_{n}\right) ; \bar{A}\right) \leq 3 c_{0}\|a\|_{A_{0}} .
$$

For the elements $u_{i}, 1 \leq i \leq n$, by (4.1), we have the estimates

$$
\begin{aligned}
\left\|u_{i}\right\|_{A_{0}} & =\left\|a_{0}\left(\tau_{i}\right)-a_{0}\left(\tau_{i-1}\right)\right\|_{A_{0}} \leq c_{0}\left\|a_{0}\left(\tau_{i}\right)\right\|_{A_{0}}+c_{0}\left\|a_{0}\left(\tau_{i-1}\right)\right\|_{A_{0}} \\
& \leq 2 c_{0} K\left(\tau_{i}, a ; \bar{A}\right)+2 c_{0} K\left(\tau_{i-1}, a ; \bar{A}\right)
\end{aligned}
$$

and so

$$
\left\|u_{i}\right\|_{A_{0}} \leq 4 c_{0} K\left(\tau_{i}, a ; \bar{A}\right)
$$

Also

$$
\begin{aligned}
\left\|u_{i}\right\|_{A_{1}} & =\left\|a_{0}\left(\tau_{i}\right)-a_{0}\left(\tau_{i-1}\right)\right\|_{A_{1}} \leq c_{1}\left\|a-a_{0}\left(\tau_{i}\right)\right\|_{A_{1}}+c_{1}\left\|a-a_{0}\left(\tau_{i-1}\right)\right\|_{A_{1}} \\
& \leq \frac{2 c_{1} K\left(\tau_{i}, a ; \bar{A}\right)}{\tau_{i}}+\frac{2 c_{1} K\left(\tau_{i-1}, a ; \bar{A}\right)}{\tau_{i-1}}
\end{aligned}
$$

and so

$$
\left\|u_{i}\right\|_{A_{1}} \leq 4 c_{1} \frac{K\left(\tau_{i-1}, a ; \bar{A}\right)}{\tau_{i-1}} .
$$

Let us see that for all $t>0$,

$$
\left(\sum_{i=1}^{n} \min \left(\left\|u_{i}\right\|_{A_{0}}^{q}, t^{q}\left\|u_{i}\right\|_{A_{1}}^{q}\right)\right)^{1 / q} \leq c K(t, a ; \bar{A})
$$

where $c$ depends only on $q, c_{0}$ and $c_{1}$. There are three cases:

1. $t<\tau_{0}$

2. For some $0 \leq k \leq n-1$ we have $\tau_{k} \leq t<\tau_{k+1}$;

3. $t>\tau_{n}$. 
We consider the second case. We have two subcases: $\tau_{k+1}=\tau_{k+1}^{+}$and $\tau_{k+1}=\tau_{k+1}^{-}$.

If $\tau_{k+1}=\tau_{k+1}^{+}$then, by $(4.5)$,

$$
K\left(\tau_{k+1}, a ; \bar{A}\right)=2 K\left(\tau_{k}, a ; \bar{A}\right) \leq 2 K(t, a ; \bar{A}) .
$$

By (4.10) and (4.11),

$$
\begin{aligned}
\sum_{i=1}^{n} \min \left(\left\|u_{i}\right\|_{A_{0}}^{q}, t^{q}\left\|u_{i}\right\|_{A_{1}}^{q}\right) & \\
& \leq \sum_{i \leq k+1}\left\|u_{i}\right\|_{A_{0}}^{q}+t^{q} \sum_{i>k+1}\left\|u_{i}\right\|_{A_{1}}^{q} \\
& \leq\left(4 c_{0}\right)^{q} \sum_{i \leq k+1} K^{q}\left(\tau_{i}, a ; \bar{A}\right)+\left(4 c_{1}\right)^{q} t^{q} \sum_{i>k+1} \frac{K^{q}\left(\tau_{i-1}, a ; \bar{A}\right)}{\tau_{i-1}^{q}} \\
& =\left(4 c_{0}\right)^{q} K^{q}\left(\tau_{k+1}, a ; \bar{A}\right)+\left(4 c_{0}\right)^{q} \sum_{i \leq k} K^{q}\left(\tau_{i}, a ; \bar{A}\right) \\
& +\left(4 c_{1}\right)^{q} t^{q} \sum_{i>k+1} \frac{K^{q}\left(\tau_{i-1}, a ; \bar{A}\right)}{\tau_{i-1}^{q}} .
\end{aligned}
$$

By (4.6) and (4.8),

$$
\begin{aligned}
& \sum_{i \leq k} K^{q}\left(\tau_{i}, a ; \bar{A}\right)+t^{q} \sum_{i>k} \frac{K^{q}\left(\tau_{i}, a ; \bar{A}\right)}{\tau_{i}^{q}} \\
& \quad \leq \sum_{i=0}^{k} 2^{q(i-k)} K^{q}\left(\tau_{k}, a ; \bar{A}\right)+t^{q} \sum_{i=k+1}^{n} 2^{q(k+1-i)} \frac{K^{q}\left(\tau_{k+1}, a ; \bar{A}\right)}{\tau_{k+1}^{q}} \\
& \quad \leq c\left(K^{q}\left(\tau_{k}, a ; \bar{A}\right)+t^{q} \frac{K^{q}\left(\tau_{k+1}, a ; \bar{A}\right)}{\tau_{k+1}^{q}}\right) \\
& \quad \leq c\left(K^{q}(t, a ; \bar{A})+t^{q} \frac{K^{q}(t, a ; \bar{A})}{t^{q}}\right) \leq c K^{q}(t, a ; \bar{A})
\end{aligned}
$$

so that by (4.12),

$$
\sum_{i=1}^{n} \min \left(\left\|u_{i}\right\|_{A_{0}}^{q}, t^{q}\left\|u_{i}\right\|_{A_{1}}^{q}\right) \leq c K^{q}(t, a ; \bar{A})
$$

where $c=c\left(q, c_{0}, c_{1}\right)$. In the second subcase, $\tau_{k+1}=\tau_{k+1}^{-}$, and so, by (4.8),

$$
\frac{K\left(\tau_{k}, a ; \bar{A}\right)}{2 \tau_{k}}=\frac{K\left(\tau_{k+1}^{-}, a ; \bar{A}\right)}{\tau_{k+1}^{-}}=\frac{K\left(\tau_{k+1}, a ; \bar{A}\right)}{\tau_{k+1}} \leq \frac{K(t, a ; \bar{A})}{t} .
$$


We write

$$
\begin{aligned}
\sum_{i=1}^{n} \min \left(\left\|u_{i}\right\|_{A_{0}}^{q},\right. & \left.t^{q}\left\|u_{i}\right\|_{A_{1}}^{q}\right) \\
\leq & \left(4 c_{0}\right)^{q} \sum_{i \leq k} K^{q}\left(\tau_{i}, a ; \bar{A}\right)+\left(4 c_{1}\right)^{q} t^{q} \sum_{i>k} \frac{K^{q}\left(\tau_{i-1}, a ; \bar{A}\right)}{\tau_{i-1}^{q}} \\
= & \left(4 c_{0}\right)^{q} \sum_{i \leq k} K^{q}\left(\tau_{i}, a ; \bar{A}\right)+\left(4 c_{1}\right)^{q} t^{q} \frac{K^{q}\left(\tau_{k}, a ; \bar{A}\right)}{\tau_{k}^{q}} \\
& +\left(4 c_{1}\right)^{q} t^{q} \sum_{i>k+1} \frac{K^{q}\left(\tau_{i-1}, a ; \bar{A}\right)}{\tau_{i-1}^{q}} \\
\leq & \left(4 c_{0}\right)^{q} \sum_{i \leq k} K^{q}\left(\tau_{i}, a ; \bar{A}\right)+\left(8 c_{1}\right)^{q} t^{q} \frac{K^{q}(t, a ; \bar{A})}{t^{q}} \\
& +\left(4 c_{1}\right)^{q} t^{q} \sum_{i>k+1} \frac{K^{q}\left(\tau_{i-1}, a ; \bar{A}\right)}{\tau_{i-1}^{q}}
\end{aligned}
$$

Repeating the calculation in (4.13) we have

$$
\begin{aligned}
\sum_{i=1}^{n} \min \left(\left\|u_{i}\right\|_{A_{0}}^{q}, t^{q}\left\|u_{i}\right\|_{A_{1}}^{q}\right) \leq & \left(4 c_{0}\right)^{q} \sum_{i=0}^{k} 2^{q(i-k)} K^{q}\left(\tau_{k}, a ; \bar{A}\right)+\left(8 c_{1}\right)^{q} K^{q}(t, a ; \bar{A}) \\
& +\left(4 c_{1}\right)^{q} t^{q} \sum_{i=k+1}^{n} 2^{q(k+1-i)} \frac{K^{q}\left(\tau_{k+1}, a ; \bar{A}\right)}{\tau_{k+1}^{q}} \\
\leq & c K^{q}(t, a ; \bar{A}) .
\end{aligned}
$$

Consider the third case, $\tau_{n}<t$. By (4.7),

$$
K\left(\tau_{n}, a ; \bar{A}\right) \leq 2 K\left(\tau_{n-1}, a ; \bar{A}\right) \leq 2 K(t, a ; \bar{A}),
$$

and by (4.10),

$$
\begin{aligned}
\sum_{i=1}^{n} \min \left(\left\|u_{i}\right\|_{A_{0}}^{q}, t^{q}\left\|u_{i}\right\|_{A_{1}}^{q}\right) & \leq \sum_{i=1}^{n}\left\|u_{i}\right\|_{A_{0}}^{q} \leq\left(4 c_{0}\right)^{q} \sum_{i=1}^{n} K^{q}\left(\tau_{i}, a ; \bar{A}\right) \\
& \leq\left(8 c_{0}\right)^{q} K^{q}(t, a ; \bar{A})+\left(4 c_{0}\right)^{q} \sum_{i=1}^{n-1} K^{q}\left(\tau_{i}, a ; \bar{A}\right) \\
& \leq c K^{q}(t, a ; \bar{A}) .
\end{aligned}
$$

Finally, we consider the first case, $t<\tau_{0}$. By (4.11),

$$
\begin{aligned}
\sum_{i=1}^{n} \min \left(\left\|u_{i}\right\|_{A_{0}}^{q}, t^{q}\left\|u_{i}\right\|_{A_{1}}^{q}\right) & \leq t^{q} \sum_{i=1}^{n}\left\|u_{i}\right\|_{A_{1}}^{q} \leq\left(4 c_{1}\right)^{q} t^{q} \sum_{i=1}^{n} \frac{K^{q}\left(\tau_{i-1}, a ; \bar{A}\right)}{\tau_{i-1}^{q}} \\
& \leq c K^{q}(t, a ; \bar{A}) .
\end{aligned}
$$


To get the claim of the theorem we take $t_{0}=\tau_{0}, t_{\infty}=\tau_{n}$ and for $1 \leq i \leq n$,

$$
t_{i}= \begin{cases}\left\|u_{i}\right\|_{A_{0}} /\left\|u_{i}\right\|_{A_{1}} & \text { if } u_{i} \neq 0 \\ 1 & \text { if } u_{i}=0\end{cases}
$$

We then have

$$
\min \left(\left\|u_{i}\right\|_{A_{0}}, t\left\|u_{i}\right\|_{A_{1}}\right)=\min \left\{1, t / t_{i}\right\} J\left(t_{i}, u_{i} ; \bar{A}\right)
$$

and, together with (4.4) and (4.9), we obtain (2.17).

\section{References}

[1] J. Bergh and J. Löfström, Interpolation Spaces. An Introduction, Springer, 1976.

[2] Yu. A. Brudnyi and N. Ya. Krugljak, Interpolation Functors and Interpolation Spaces, North-Holland, Amsterdam, 1991.

[3] M. Cwikel, $K$-divisibility of the $K$-functional and Calderón couples, Ark. Mat. 22 (1984), 39-62.

[4] B. Jawerth and M. Milman, Extrapolation theory with applications, Mem. Amer. Math. Soc. 89 (1991), no. 440.

[5] P. Nilsson, Reiteration theorems for real interpolation and approximation spaces, Ann. Mat. Pura Appl. (4) 132 (1982), 291-330.

[6] J. Peetre and G. Sparr, Interpolation of normed Abelian groups, ibid. (4) 92 (1972), 217-262.

[7] R. T. Rockafellar, Convex Analysis, Princeton Univ. Press, Princeton, 1970.

[8] Y. Sagher, A new interpolation theorem, in: Lecture Notes in Math. 908, Springer, 1982, 189-198.

[9] Y. Sagher and P. Shvartsman, An interpolation theorem with perturbed continuity, J. Funct. Anal. 188 ( 2002) , 75-110.

[10] - - - The approximation functional and interpolation with perturbed continuity, J. Approx. Theory 110 (2001) , 236-260.

[11] - - - Rearrangement-function inequalities and interpolation theory, ibid. 119 (2002), 214-251.

Department of Mathematics

Luleå University of Technology

SE-971 87 Luleå, Sweden

E-mail: natan@sm.luth.se

Department of Mathematics

Technion-Israel Institute of Technology

32000 Haifa, Israel

E-mail: pshv@tx.technion.ac.il
Department of Mathematical Sciences

Florida Atlantic University Boca Raton, FL 33431-0991, U.S.A.

E-mail: sagher@fau.edu

Received December 15, 2003

Revised version April 12, 2005 